\title{
Use and valuation of native and introduced medicinal plant species in Campo Hermoso and Zetaquira, Boyacá, Colombia
}

Ana Lucía Cadena-González ${ }^{1 *}$, Marten Sørensen² and Ida Theilade ${ }^{3}$

\begin{abstract}
Background: Medicinal plant species contribute significantly to folk medicine in Colombia. However, few local studies have investigated whether species used are introduced or native and whether there is a difference in importance of native and introduced medicinal plant species. The aim of the present study was to describe the use of medicinal plants within two municipalities, Campo Hermoso and Zetaquira, both in the department of Boyacá, Colombia and to assess the importance of native and introduced plants to healers, amateur healers and local people. As local healers including amateur healers have no history of introduced species our working hypotheses $\left(\mathrm{H}_{1-2}\right)$ were that $\mathrm{H}_{1}$ : native and introduced medicinal plant species are of equal importance and $\mathrm{H}_{2}$ : healers and amateur healers do not differentiate in their preferences between native and introduced medicinal plant species.
\end{abstract}

Methods: Ten villages were included in the study. A combination of quantitative and qualitative methods was used including questionnaires, semi-structured interviews, in- depth interviews, and open talks. Voucher specimens were collected in home gardens and during field walks. For data analysis, we calculated use value indices and Jaccard index and tested for the above hypothesis using Spearman rank-correlation coefficients and Wilcoxon-Mann-Whitney tests.

Results: Eighty medicinal plant species were described by locals as the most frequently used. Of these, 78 species were taxonomically identified, distributed within 41 families and 74 genera, which included 35 native species and 43 introduced. The highest valued families were: Asteraceae, Lamiaceae, Apiaceae, Rutaceae and Verbenaceae. The species ranked highest according to their Use Values, in both municipalities, were Mentha suaveolens Ehrh., Ambrosia cumanensis Kunth, and Verbena littoralis Kunth. Introduced species were more important than native ones in Zetaquira, while there was no difference in importance in Campo Hermoso. While healers relied most on the uses of native species, amateur healers were inclined to rely on introduced species. Medicinal plant administration in both municipalities follow the usual pattern: Leaves are used most commonly prepared by decoction or infusion and administrated orally.

Conclusions: The high proportion of introduced plant species used in the local traditional medicines is similar to the results of a number of other ethnobotanical studies and emphasise the need for efforts to record and maintain traditional knowledge on native species.

Keywords: Acculturation, Ethnobotany, Trained healers, Amateur healers, Introduced medicinal plants, Traditional knowledge

\footnotetext{
* Correspondence: analu@dsr.life.ku.dk

1 Department of Agriculture and Ecology, University of Copenhagen,

Rolighedsvej 21, 1958 Frederiksberg C, Denmark

Full list of author information is available at the end of the article
} 


\section{Background}

Medicinal plant species constitute important alternatives to conventional medicine in a large number of developing countries, especially within poor communities that inhabit rural areas and lack access to health services [1,2]. A number of native medicinal plants of both the palaeotropics and neotropics have traditionally had a high value for indigenous communities, not only because of their healing properties, but also due to other uses [3]. An example of such multi-use plants is the neotropical species Crescentia cujete L., a source of both timber and traditional medicine. Recently, this species has reached the United States and European pharmaceutical markets demonstrating that traditional medicinal plants used by indigenous communities may be of high economic value [3].

However, knowledge, cultivation and maintenance of these native species within rural communities is decreasing, due to modernization processes, such as acculturation [1-3]. In addition, a tendency to consider all plant resources as native by local people has been directly or indirectly documented in several studies [4-6]; in other words, numerous introduced plant species with healing properties have become popularly considered as 'native'. Consequently, a number of native medicinal plant species have been replaced by introduced species. Thus, they use the terms 'native' and 'introduced' indiscriminately so that many introduced species with healing properties have become popularly known as native. For these reasons, the uses of a number of native species have been replaced by introduced species, incorrectly perceived as native.

When faced with the rapid decline in traditional knowledge it is relevant to identify medicinal species and to record their uses within local communities. This is especially important in regions that currently are affected by land-use change and modernisation. Documentation and awareness of ethnobotanical knowledge within these regions may facilitate the maintenance of medicinal plant resources and uses.

Furthermore, ethnobotanical studies may support implementation of strategies that integrate native medicinal plant uses with sustainable management of natural resources. The municipalities of Campo Hermoso and Zetaquira in the department of Boyacá, Colombia, are examples where conversion of forest areas into agriculture land and reforestation with exotic species have destroyed large areas of natural forest including high plateau, upland, and montane forest. Furthermore, many multiple-use plants have been overexploited $[7,8]$. This situation has caused loss of a number of native species (pers. comm. local key informants 2009). Studies on medicinal plant use have been conducted in different localities within Boyacá $[9,10]$, but none in the regions of
Campo Hermoso and Zetaquira. Therefore, the present study was carried out within these municipalities. The aims of this study were to identify the medicinal plant species, to determine their origin (native or introduced to Colombia), to record their medicinal uses (targeted illnesses, ways of administration), and based on personal observations and inspired by previous studies [4-6] to assess the value of native and introduced plant species to members of local communities. The latter objective was evaluated by the null hypotheses $\left(\mathrm{H}_{1-2}\right) \mathrm{H}_{1}$ : native and introduced medicinal plant species are of equal importance and $\mathrm{H}_{2}$ : healers and amateur healers do not differentiate in their preferences between native and introduced medicinal plant species.

\section{Methods}

\section{Study areas}

The study took place in the municipalities of Campo Hermoso and Zetaquira in department of Boyacá, Colombia (Figure 1). The municipalities are located on the Eastern slope of the Cordillera Oriental (Eastern Andean range) and their terrain comprises rugged areas with peaks, steep canyons, and valleys. Both study areas belong to the humid subtropical zone $[7,8]$. The altitude range from 500-2500 m a.s.l. in Campo Hermoso and $1875-3600 \mathrm{~m}$ a.s.l. in Zetaquira. Temperatures range between $12-35^{\circ} \mathrm{C}$.

Climatic differences and mountainous topography lead to a diversity of natural vegetation, livestock pastures, and crops within the study areas $[7,8]$. Campo Hermoso is located at $132 \mathrm{~km}$ from Tunja the capital of the department and $143 \mathrm{~km}$ from Bogotá. Zetaquira is located at $69 \mathrm{~km}$ away from Tunja, and at $126 \mathrm{~km}$ away from Bogotá.

The economy of the municipalities is based on cattle ranching and agriculture; the latter often by smallholders practicing subsistence economy. The main products in Zetaquira are coffee, beans, sugarcane, maize, plantain, manioc and 'arracacha' (Arracacia xanthorrhiza Bancr.) [11]. The coffee production is located near the villages at intermediate altitudes. Until 2008, coffee was a main crop in Zetaquira. However, climate change has decreased feasibility of coffee production in recent years (pers. comm.: Domingo Mendoza, responsible of the Federación Nacional de Cafeteros de Colombia, office in Zetaquira). Campo Hermoso is basically a cattle ranching region with large areas of pastures [7]. Besides, maize, beans, manioc and vegetables and, at smaller scale, sugarcane is cultivated for subsistence. There are no conspicuous economic differences between the two municipalities $[7,8]$.

Data collection and free, prior informed consent (FPIC) The field work was conducted from October 2008 to January 2009. Data collection included a combination of 


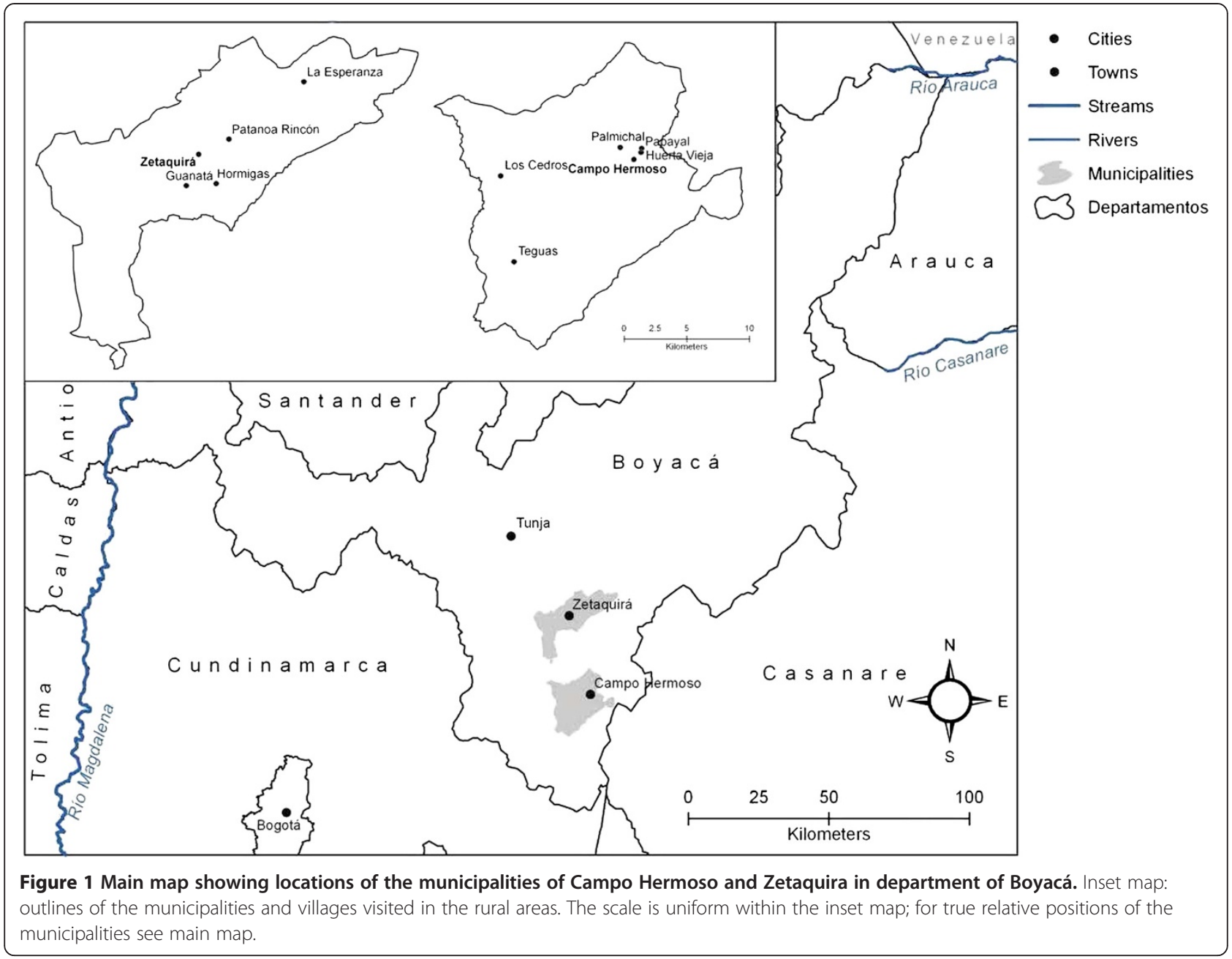

quantitative and qualitative methods in order to allow for triangulating and cross-checking [12]. Applied and complementary ethnobotanical methods [13] were used to acquire information on how local people related to the medicinal plant resources in the two municipalities. The project was presented to the local authorities and to the communities during initial meetings. Objectives and methods were approved in both municipalities.

\section{Selection of villages and identification of informants}

Within each municipality representative villages were visited. Ten villages were chosen according to distance to the main urban centre, the presence of a representative flora, and residential areas of the healers and herbalists, who were purposefully included in the sampling. In Zetaquira the villages studied were: La Esperanza, Guanata, Centro Rural and Hormigas; and in Campo Hermoso: Papayal, Huerta Vieja, Palmichal, Los Cedros, Macanalito and Teguas.

Participation of local people was essential to several stages of the research process [12]. Thus, key informants in the municipalities were contacted in the first stage. Their collaboration was crucial for selecting and planning the visits to the villages.

Each municipality was visited three times during the study in order to establish and maintain communication with the local informants, to identify additional informants, and later to apply quantitative and qualitative tools. The informants from the villages were selected using stratified random sampling. Interviewees in the study all belonged to farmers' communities.

\section{Application of questionnaires, in-depth interviews and semi- structured interviews}

Questionnaires were designed to collect information on the main local medicinal plant uses. The questions were answered by a selected group $(n=9)$ of old women in Patanoa Rincon in Zetaquira. Interviews were structured in two groups: in depth interviews and semi-structured interviews, which were done with individual informants in order to maintain data independence [13]. In depth interviews were answered by healers ${ }^{\mathrm{a}}$ and amateur 
healers ${ }^{\mathrm{b}}$. In-depth interviews were carried out in order to collect information on their knowledge on plant use and their services offered to locals. Semi-structured interviews addressed farmers $(n=41)$. Their distribution across age and sex is shown in Table 1. Semi-structured interviews helped to collect data on traditional plant knowledge and use. For all interviews, vouchers of local medicinal plant species was used as a stimulus for interviewees to elaborate on known and used plants [14].

Homegardens, gardens and solares ${ }^{\mathrm{c}}$ were visited during the interviews. Plant specimens were collected together with the informants when obtainable. From the list of mentioned plants, those that were not cultivated at home were grouped in a separate list.

Disease was defined by interviewees as a period of disequilibrium in the body due to deterioration of organs and functions. Disequilibrium could also be caused by bacteria and viral infection. The diseases described during the study were grouped into categories as it is shown in Table 2.

\section{Field walks}

A field walk with key informants was conducted in each municipality in order to identify environmental characteristics of medicinal plant species. The field walk in Campo Hermoso took place along a $5 \mathrm{~km}$ transect. In Zetaquira, it was conducted along a $4.5 \mathrm{~km}$ transect. Both transects traversed hills and sloping terrain, agricultural lands, and pastures for cattle ranching with some spots of secondary forests.

\section{Systematics of plant species}

Taxonomic identification was mainly conducted by experts at the Colombian National Herbarium (COL) and at the herbarium of Universidad Tecnológica de Tunja and, the Colombian Amazonian Herbarium (COAH) [15]. Collected specimens were deposited at COL, at the non-profit organization Alexander von Humboldt Biological Resources Research Institute Villa de Leyva branch, Colombia (FMB), and at the Herbarium, Botany Group, Department of Plant and Environmental Sciences, Faculty of Science, University of Copenhagen (CP), for future references. Additionally, specimens were

Table 1 Distribution of informants who answered semistructured interviews in the municipalities of Campo Hermoso and Zetaquira, including totals, percentage of interviewees native to the municipality, and age classes

\begin{tabular}{lllllll}
\hline & & & \multicolumn{3}{c}{ Age class } \\
\cline { 4 - 6 } Municipality & Total & \% native & $\mathbf{2 0}$ to $\mathbf{4 0}$ & $\mathbf{4 1}$ to $\mathbf{6 0}$ & $\mathbf{7 0}$ \\
\hline Campo Hermoso & 16 & 81 & 1 & 7 & 8 \\
Zetaquira & 25 & 80 & 6 & 11 & 8 \\
\hline
\end{tabular}

deposited with the local authorities of each municipality, for reference and further studies at the local level.

\section{Data analysis}

Different measures of knowledge described by interviewees can be seen in ethnobotanical research. Therefore, it was decided to carefully evaluate the measure of knowledge to be used in the data analysis of the present study.

Table 2 Categories of diseases with the respective descriptions recorded in the municipalities of Zetaquira and Campo Hermoso

\begin{tabular}{ll}
\hline Category & Diseases \\
\hline Digestive system & Inflamations of intestine, intestinal \\
& obstipation, intestinal obstipation in \\
& newborns, diarrhoea, apendicitis, stomach \\
& aches, enhance stomach function, gastritis, \\
& stomach ulcer, against stomach acids, liver \\
& cleanser, hepatitis, against bad breath, \\
& againts umbilical, hernia, diabetes, high \\
& bilirubin in new born, tooth pain, mouth \\
& driedness, sensitive gums
\end{tabular}

Bacterial,parasitic and mycotic infections

Musculoskeletal system

Nervous system

Inmune system

Integumentary system

Reproductive system

Respiratory system

Urinary system

Cancer

Accidents

Specific conditions
Inflamations and skin infections, erisipela, againts acne and feet's fungus, against warts, infections in throat, gripe, intestinal parasits, eye inflamations and infections, eye dust, mouth infections, againts hair lice, typhus, denge

Rheumatism, colds and spams in muscles and tendons, fractures in bones, back pain

Memory loss, nervous system tonics, nervous tension, children with problems to fall asleep tiredness and weakness, body dryness

Fevers, yellow fever

External and internal inflamations, allergies, infected wounds, excema, spots, rashes in adults, diaper rash

Prostata complaints, weak uterus, cramps in uterus and menstrual pains, uterus cleanser, menstrual disorders, healing of uterus after giving birth, to prevent extra bleeding during child delivery, to enhance lactation

Cardiac problems as hypertrophy, hipertension complaints, blood cleanser and tonic, antihemorragic, to stop nose bleeding in children

Sinusitis, to enhance lungs function, asthma, coughs

Children bladder complaints, kidney and bladder complaints in adults

Stomach cancer, skin cancer, cancer in general

Ofidic accidents

Headaches, general indisposition, againts flies, to lose weight, hidropisy, againts hair loss, hair treatments

Other

Food, condiment herb 
Plant species mentioned by the informants could be associated with one or several diseases, with one or more uses, or with both diseases and uses. Hence, it was possible to sum up two-way combinations of plant species (with use or disease) or three-way combinations (with use and disease). Since species often were only associated with one or the other, the different combinations might relate to substantially different measures of knowledge. Further, native and introduced species were distinguished, so that it was possible to observe differences of use between the two groups of plant species.

Spearman rank-correlation coefficients $[16,17]$ of the different indices of interviewees' knowledge were calculated (Table 3). As the knowledge indices were highly correlated, (correlation coefficient 0.87 ) it was decided to use plant-disease-use combinations, because they appeared to be the most comprehensive measure.

In order to assess the interviewees' valuation of the medicinal plant species, use-value indices (UV) [18] were calculated as follows: The plant-disease-use combinations mentioned by the informants were counted. Use value (UV) was calculated by the formula [19]:

$$
U V=\frac{U}{n}
$$

where $U=$ plant-disease-use combination, and $n=$ total number of interviewees in each municipality. Since the informants were interviewed only once, it was decided to interpret each plant-disease-use combination mentioned by each informant as an event.

Furthermore, estimated UVs and actual UVs were calculated for each species following the procedure described above.

In addition, estimated and actual Family Use Values (FUV) were calculated in order to identify the significance of medicinal plant families in the municipalities. For this purpose, Equation 1 was adapted in accordance with the literature [20]:

$$
F U V=\frac{U V s}{(n s)}
$$

where $U V s=$ use values of the species, and $n s=$ total number of species within each family.

Table 3 Spearman rank correlations of different variants of knowledge indices. Correlation coefficients are given

\begin{tabular}{|c|c|c|c|c|c|c|c|c|c|c|c|}
\hline & $\begin{array}{l}\text { Plant- } \\
\text { Disease-Use- } \\
\text { Comb. total }\end{array}$ & $\begin{array}{l}\text { Plant- } \\
\text { Disease-Use- } \\
\text { Comb. native }\end{array}$ & $\begin{array}{l}\text { Plant- } \\
\text { Disease- } \\
\text { Comb. } \\
\text { total }\end{array}$ & $\begin{array}{l}\text { Plant- } \\
\text { Disease- } \\
\text { Comb. } \\
\text { native }\end{array}$ & $\begin{array}{l}\text { Plant- } \\
\text { Use- } \\
\text { Comb. } \\
\text { total }\end{array}$ & $\begin{array}{l}\text { Plant- } \\
\text { Use- } \\
\text { Comb. } \\
\text { native }\end{array}$ & $\begin{array}{l}\text { Plants } \\
\text { mentioned }\end{array}$ & $\begin{array}{l}\text { Plant } \\
\text { with } \\
\text { use }\end{array}$ & $\begin{array}{l}\text { Plants } \\
\text { at } \\
\text { home }\end{array}$ & $\begin{array}{l}\text { Total } \\
\text { no. of } \\
\text { uses }\end{array}$ & $\begin{array}{l}\text { Total } \\
\text { no. of } \\
\text { diseases }\end{array}$ \\
\hline $\begin{array}{l}\text { Plant- } \\
\text { Disease-Use- } \\
\text { Comb. total }\end{array}$ & & 0.87 & 0.97 & 0.84 & 0.97 & 0.81 & 0.92 & 0.94 & 0.69 & 0.85 & 0.82 \\
\hline $\begin{array}{l}\text { Plant- } \\
\text { Disease-Use- } \\
\text { Comb. native }\end{array}$ & $<0.001$ & & 0.85 & 0.94 & 0.83 & 0.94 & 0.80 & 0.85 & 0.55 & 0.82 & 0.76 \\
\hline $\begin{array}{l}\text { Plant- } \\
\text { Disease- } \\
\text { Comb. total }\end{array}$ & $<0.001$ & $<0.001$ & & 0.87 & 0.96 & 0.81 & 0.93 & 0.95 & 0.72 & 0.83 & 0.81 \\
\hline $\begin{array}{l}\text { Plant- } \\
\text { Disease- } \\
\text { Comb. native }\end{array}$ & $<0.001$ & $<0.001$ & $<0.001$ & & 0.81 & 0.88 & 0.79 & 0.83 & 0.55 & 0.81 & 0.82 \\
\hline $\begin{array}{l}\text { Plant-Use- } \\
\text { Comb. total }\end{array}$ & $<0.001$ & $<0.001$ & $<0.001$ & $<0.001$ & & 0.85 & 0.94 & 0.95 & 0.70 & 0.83 & 0.80 \\
\hline $\begin{array}{l}\text { Plant-Use- } \\
\text { Comb. native }\end{array}$ & $<0.001$ & $<0.001$ & $<0.001$ & $<0.001$ & $<0.001$ & & 0.80 & 0.82 & 0.52 & 0.78 & 0.70 \\
\hline $\begin{array}{l}\text { Plants } \\
\text { mentioned }\end{array}$ & $<0.001$ & $<0.001$ & $<0.001$ & $<0.001$ & $<0.001$ & $<0.001$ & & 0.95 & 0.76 & 0.82 & 0.78 \\
\hline $\begin{array}{l}\text { Plant with } \\
\text { use }\end{array}$ & $<0.001$ & $<0.001$ & $<0.001$ & $<0.001$ & $<0.001$ & $<0.001$ & $<0.001$ & & 0.74 & 0.84 & 0.78 \\
\hline $\begin{array}{l}\text { Plants at } \\
\text { home }\end{array}$ & $<0.001$ & $<0.001$ & $<0.001$ & $<0.001$ & $<0.001$ & $<0.001$ & $<0.001$ & $\begin{array}{l}< \\
0.001\end{array}$ & & 0.59 & 0.57 \\
\hline $\begin{array}{l}\text { Total no. of } \\
\text { uses }\end{array}$ & $<0.001$ & $<0.001$ & $<0.001$ & $<0.001$ & $<0.001$ & $<0.001$ & $<0.001$ & $\begin{array}{l}< \\
0.001\end{array}$ & $\begin{array}{l}< \\
0.001\end{array}$ & & 0.84 \\
\hline $\begin{array}{l}\text { Total no. of } \\
\text { diseases }\end{array}$ & $<0.001$ & $<0.001$ & $<0.001$ & $<0.001$ & $<0.001$ & $<0.001$ & $<0.001$ & $\begin{array}{l}< \\
0.001\end{array}$ & $\begin{array}{l}< \\
0.001\end{array}$ & $\begin{array}{l}< \\
0.001\end{array}$ & \\
\hline
\end{tabular}
above the diagonal; corresponding $\mathrm{p}$-values below the diagonal 
Table 4 List of medicinal plant species reported in Zetaquira and Campo Hermoso including ethnobotanical characteristics

\section{Index Use}

Values (estim./

actual)

Species, family, voucher

Status, place of

Zeta- Campo

\section{ID, and vernacular Habitat/ place}

Origin quira Herm.

Justicia filibracteolata of collection

Justicia filibracteolata
Lindau Acanthaceae shrub cultivated native Colombian

ALCG 62 Chuchuhuaza

$\begin{array}{llll}\text { shrub cultivated } & \text { native Colombian } & 0.48 / & 0.06 / \\ \text { in homegarden } & \text { Andes }^{3} & 0.04 & 0.00\end{array}$

\section{Part Used}

Use

Preparation, mode of administration

Trichanthera gigantea

(Bonpl.) Nees (s)

small tree wild native to Northern

in edge of route South America

$0.32 / \quad 0.19 /$

$0.20 \quad 0.00$

leaves and stems (B)

Acanthaceae ALCG 125

Sambucus nigra L.* o

tree cultivated in introduced Europe-

Adoxaceae-Caprifoliaceae solar West Siberia-North

${ }^{++}$ALCG 68 Sauco Elder Africa $^{5}$

or Elderberry

leaves and flowers $(\mathrm{B})^{* 2}$ flowers $(B)^{2}$ leaves and branches (B) flowers and branches (B)

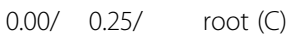

Agavaceae Fique,cabuya

Fique

Chenopodium

ambrosioides L. (s)

Amaranthaceae ALCG 80

Paico Epazote Wormseed

Althernanthera lanceolata

(Benth.) Schinz

Amaranthaceae ALCG

113 Planta del cáncer or

Sanguinaria

Eryngium foetidum L.*
$0.00 \quad 0.13$

leaves (C)

succulent rosette native Boyaca-

forming tall he
cultivated in a

farmland

herb ascendent native Central

cultivated in

Andean range

south of western

range in Colombia

herb cultivated

in small garden

introduced American

tropics and

subtropics, Asia,

Africa and Australia ${ }^{12}$

$0.40 / \quad 0.44 / \quad$ leaves (B) leaves and

$0.12 \quad 0.38$

stems (B)

$0.16 / \quad 0.48 /$

$0.16 \quad 0.25$

herb cultivated native South Mexico $0.00 / \quad 0.06 /$

in homegarden leaves (C) leaves (Z)

$\operatorname{root}(C)$ leaves and branches (B) rheumatism (B)

flammations and skin infections (B) fevers (Z) to lose weight (Z)

gripe (B) eye inflammations and infections (B) fevers (B) tiredness and weakness (Z) respiratory complaints (B) respiratory complaints (Z) headache (Z) against hair lice (C) complaints (C) Hidropisy (C)

stomach complaints (B) intestina parasites (B)

healing of wounds (B) skin cancer (C) stomach ulcer (C) cancer in general (C) cancer in general (Z) fevers (Z)

hepatitis (C) condiment herb (C) concoction with Allium sativum and

Aristolochia ringens in extract in alcohol/ oral -U1- (Z), concoction with Cannabis sativa in extract in alcohol -U2-/ oral (B), decoction/ oral (C)

decoction/topical-baths (B) decoction/ topical-baths (Z); fresh extract by maceration/ oral (Z) infusion/ oral (Z)

concoction with Citrus limon juice in decoction/ oral (B) extract in decoction or destilation (in a glass bottle)/ topical- eye baths (B) decoction/ topical in baths (B) decoction/ topical, baths (Z) decoction/ oral (B) concoction with milk in decoction/ oral (Z) decoction/ oral (B) decoction/ oral (Z) fresh extract from maceration/ topical on the head before the sunrise starts (Z) fresh extract from maceration/ topical for washing the hair (C)

decoction/ oral (C) use of fibres of leaves to tie them on the affected part/ topical (C) decoction of a piece of leaf/ oral (C)

infusion or decoction/ oral (B) fresh extract by maceration in water/ oral (B)

decoction/ oral and decoction/ topical-baths (B) decoction/ topical baths (C) decoction in milk/ oral (C) decoction / oral (C) fresh extract by maceration in water/ oral (Z)

decoction/ oral (C) combination with food/ oral (C) 
Table 4 List of medicinal plant species reported in Zetaquira and Campo Hermoso including ethnobotanical characteristics (Continued)

\begin{tabular}{|c|c|c|c|c|c|c|c|}
\hline Apiaceae & & \multirow{4}{*}{$\begin{array}{l}\text { Andeans and } \\
\text { Orinoquia }^{5}\end{array}$} & & & \multirow[t]{4}{*}{ leaves $(C) * 2$} & & \\
\hline ALCG 167 & & & & & & & \\
\hline \multicolumn{6}{|l|}{$\begin{array}{l}\text { Cilantrón,cilantro } \\
\text { cimarrón }\end{array}$} & & \\
\hline \multicolumn{6}{|l|}{$\begin{array}{l}\text { Mexican coriander, } \\
\text { shadow beni }\end{array}$} & & \\
\hline Apium graveolens L. * & \multirow{6}{*}{$\begin{array}{l}\text { herb cultivated } \\
\text { in homegarden }\end{array}$} & \multirow{6}{*}{$\begin{array}{l}\text { introduced Europe } \\
\text { and West Asia }{ }^{13} \text {, } \\
\text { Europe }{ }^{5}\end{array}$} & \multirow{6}{*}{$\begin{array}{l}0.20 / \\
0.24\end{array}$} & \multirow{6}{*}{$\begin{array}{l}0.25 / \\
0.19\end{array}$} & \multirow[t]{6}{*}{ leaves and stems $(\mathrm{B})^{*} 2$} & \multirow[t]{2}{*}{ intestinal obstipation (B) } & decoction/oral(B) \\
\hline Apiaceae & & & & & & & infusion/oral(B) \\
\hline Apio & & & & & & stomach ache (B) & combination with food/oral(B) \\
\hline \multirow[t]{3}{*}{ Celery,celeriac } & & & & & & condiment herb (B) & infusion/oral(B) \\
\hline & & & & & & relaxant (B) & decoction/oral(Z) \\
\hline & & & & & & to regulate menstruation (Z) & \\
\hline Foeniculum vulgare Mill.* o & \multirow{6}{*}{$\begin{array}{l}\text { herb cultivated } \\
\text { in homegarden }\end{array}$} & \multirow{6}{*}{$\begin{array}{l}\text { introduced } \\
\text { Mediterranean } \\
\text { Europe }^{5}\end{array}$} & \multirow{6}{*}{$\begin{array}{l}0.16 / \\
0.16\end{array}$} & \multirow{6}{*}{$\begin{array}{l}0.06 / \\
0.06\end{array}$} & \multirow[t]{6}{*}{ leaves and stems (B) } & \multirow[t]{2}{*}{ to enhance lactation (B) } & \multirow{2}{*}{$\begin{array}{l}\text { decoction in milk or "aguadepanela"-U3-/ } \\
\text { oral (B) }\end{array}$} \\
\hline Apiaceae & & & & & & & \\
\hline ALCG 89 & & & & & & condiment herb (B) & combination with food/ oral (B) \\
\hline \multicolumn{3}{|l|}{ Hinojo } & & & & & \\
\hline \multicolumn{3}{|l|}{ Fennel, Bronze Fennel, } & & & & & \\
\hline \multicolumn{3}{|l|}{ Sweet cumin ${ }^{12}$} & & & & & \\
\hline \multirow{3}{*}{$\begin{array}{l}\text { Petroselinum crispum } \\
\text { (Mill.) Nyman ex A.W. Hill** }\end{array}$} & \multirow{10}{*}{$\begin{array}{l}\text { herb cultivated } \\
\text { in homegarden }\end{array}$} & \multirow{10}{*}{$\begin{array}{l}\text { introduced Eastern } \\
\text { Mediterranean } \\
\text { Western Asia } \\
\text { Mediterranean } \\
\text { Europe }^{8}\end{array}$} & \multirow{10}{*}{$\begin{array}{l}0.44 / \\
0.36\end{array}$} & \multirow{10}{*}{$\begin{array}{l}0.50 / \\
0.25\end{array}$} & \multirow[t]{10}{*}{ leaves $(B)^{*} 2$} & \multirow{3}{*}{$\begin{array}{l}\text { cardiovascular complaints as high } \\
\text { blood pressure (B) }\end{array}$} & infusion/ oral (B) \\
\hline & & & & & & & to chew leaves/ oral (B) \\
\hline & & & & & & & combined with food/ oral (B) \\
\hline Apiaceae & & & & & & enhance stomach function (B) & infusion/ oral (C) \\
\hline ALCG 87 & & & & & & & infusion/ oral (Z) \\
\hline Perejil & & & & & & againts bad breath (B) & \\
\hline \multirow[t]{4}{*}{ Parsley } & & & & & & condiment herb (B) & \\
\hline & & & & & & kidney complaints (C) & \\
\hline & & & & & & intestinal pain (C) & \\
\hline & & & & & & againts menstrual pain (Z) & \\
\hline $\begin{array}{l}\text { Catharanthus roseus (L.) G. } \\
\text { Don. }\end{array}$ & \multirow[t]{4}{*}{$\begin{array}{l}\text { short lived herb } \\
\text { cultivated in pod }\end{array}$} & $\begin{array}{l}\text { introduced } \\
\text { Madagaskar }\end{array}$ & $\begin{array}{l}0.00 / \\
0.00\end{array}$ & $\begin{array}{l}0.06 / \\
0.06\end{array}$ & flowers (C) & $\begin{array}{l}\text { againts eye infections and irritations } \\
\text { (C) }\end{array}$ & infusion/ topical-baths (C) \\
\hline Apocynaceae & & & & & & & \\
\hline ALCG 158 & & & & & & & \\
\hline Vicaria & & & & & & & \\
\hline Madagascar periwinkle & & & & & & & \\
\hline
\end{tabular}


Table 4 List of medicinal plant species reported in Zetaquira and Campo Hermoso including ethnobotanical characteristics (Continued)

\begin{tabular}{|c|c|c|c|c|c|c|c|}
\hline $\begin{array}{l}\text { Philodendron sp. } \\
\text { Araceae } \\
\text { ALCG } 103 \\
\text { Hidra or Yedra }\end{array}$ & $\begin{array}{l}\text { sufrutex wild in } \\
\text { edges of } \\
\text { secundary forest }\end{array}$ & $\begin{array}{l}\text { unknown Africa and } \\
\text { Asia and America } \\
\text { around the equador } \\
7\end{array}$ & $\begin{array}{l}0.04 / \\
0.04\end{array}$ & $\begin{array}{l}0.00 / \\
0.00\end{array}$ & leaves and stem (Z) & skin infections and inflamations (Z) & $\begin{array}{l}\text { fresh extract by maceration/ topical-rub part } \\
\text { affected }\end{array}$ \\
\hline $\begin{array}{l}\text { Colocasia esculenta (L.) } \\
\text { Schott }\end{array}$ & \multirow[t]{5}{*}{$\begin{array}{l}\text { herb cultivated } \\
\text { in homegarden }\end{array}$} & \multirow[t]{5}{*}{$\begin{array}{l}\text { introduced South } \\
\text { East Asia, Australia } \\
8,13\end{array}$} & \multirow[t]{5}{*}{$\begin{array}{l}0.20 / \\
0.16\end{array}$} & \multirow[t]{5}{*}{$\begin{array}{l}0.31 / \\
0.25\end{array}$} & \multirow[t]{5}{*}{ tuberous root (B) } & gastritis (B) & \multirow[t]{2}{*}{$\begin{array}{l}\text { concoction of blended root with milk/ oral } \\
\text { (B) }\end{array}$} \\
\hline Araceae & & & & & & stomach ulcer (B) & \\
\hline ALCG 106 & & & & & & food (B) & carbohydrate source food/ oral (B) \\
\hline \multicolumn{3}{|l|}{ Malanga } & & & & & \\
\hline \multicolumn{3}{|l|}{ Taro } & & & & & \\
\hline $\begin{array}{l}\text { Aristolochia ringens Vahl. } \\
\text { Aristolochiaceae } \\
\text { ALCG } 78 \\
\text { Bejuco de Guaco }\end{array}$ & \multirow[t]{2}{*}{$\begin{array}{l}\text { Twiner/climber } \\
\text { Wild in river } \\
\text { bank }\end{array}$} & \multirow[t]{2}{*}{$\begin{array}{l}\text { introduced native to } \\
\text { Brazil }\end{array}$} & \multirow[t]{2}{*}{$\begin{array}{l}0.64 / \\
0.04\end{array}$} & \multirow[t]{2}{*}{$\begin{array}{l}0.75 / \\
0.00\end{array}$} & \multirow[t]{2}{*}{ leaves and stems (B) } & $\begin{array}{l}\text { rheumatism (B) } \\
\text { ofidic accidents (B) }\end{array}$ & \multirow[t]{2}{*}{$\begin{array}{l}\text { concoction with Cannabis sativa, } \\
\text { Erythroxylum coca and Justicia filibracteolata } \\
\text { in extract in alcohol/ topical and oral (B), } \\
\text { maceration in extract in alcohol/ topical and } \\
\text { oral (Z)-U2- maceration/ topical in emplast } \\
\text { (B) }\end{array}$} \\
\hline Gaping Dutchman`s Pipe & & & & & & & \\
\hline $\begin{array}{l}\text { Aloe vera (L.) Burman f. * } \\
\text { Asphodelaceae } \\
\text { Zábila }\end{array}$ & \multirow[t]{7}{*}{$\begin{array}{l}\text { Short stem-med } \\
\text { suculent plant } \\
\text { cultivated in } \\
\text { pods }\end{array}$} & \multirow[t]{7}{*}{$\begin{array}{l}\text { introduced arabic } \\
\text { peninsula }^{8}\end{array}$} & \multirow[t]{7}{*}{$\begin{array}{l}0.64 / \\
0.60\end{array}$} & \multirow[t]{7}{*}{$\begin{array}{l}0.63 / \\
0.63\end{array}$} & \multirow[t]{7}{*}{$\begin{array}{l}\text { gel from leaves }(B) * 2 \circ \\
\end{array}$} & $\begin{array}{l}\text { asthma (B) } \\
\text { caugh (B) } \\
\text { fever (B) }\end{array}$ & $\begin{array}{l}\text { fresh gel is blended single or in concoction } \\
\text { with egg or with honey and juice of Citrus } \\
\text { limon/ oral (B) }\end{array}$ \\
\hline & & & & & & headache (B) & fresh gel/ topical-emplast over head-front ( $Z$ ) \\
\hline & & & & & & healing wounds and & fresh gel/ topical-emplast over head-front (B) \\
\hline \multirow{4}{*}{ Aloe } & & & & & & skin spots (B) & fresh gel/ topical-emplast over head-front (Z) \\
\hline & & & & & & external inflamations (B) & \\
\hline & & & & & & hair treatment (B) & massaging (B) \\
\hline & & & & & & stomach cancer (Z) & fresh gel in concoction with honey/ oral (Z) \\
\hline Bidens pilosa L. * & \multirow{5}{*}{$\begin{array}{l}\text { herb wild in } \\
\text { pastures with } \\
\text { bushes } \\
\text { composition }\end{array}$} & \multirow{5}{*}{$\begin{array}{l}\text { native } \text { From the } \\
\text { centrum to the south } \\
\text { of the Andeans of } \\
\text { Colombia }^{3}\end{array}$} & \multirow{5}{*}{$\begin{array}{l}0.00 / \\
0.04\end{array}$} & \multirow{5}{*}{$\begin{array}{l}0.00 / \\
0.00\end{array}$} & \multirow[t]{5}{*}{ leaves $(Z) * 2$} & skin infections ( $\mathrm{Z}$ ) rashes $(\mathrm{Z})$ & maceration/ topical-emplast (Z) \\
\hline Asteraceae & & & & & & & decoction/ topical-baths (Z) \\
\hline ALCG 144 & & & & & & & \\
\hline Chipaca & & & & & & & \\
\hline Spanish needle,black jack & & & & & & & \\
\hline $\begin{array}{l}\text { Onoseris onoseroides } \\
\text { (Kunth) Robins }\end{array}$ & $\begin{array}{l}\text { tall herb wild in } \\
\text { slope hills and }\end{array}$ & $\begin{array}{l}\text { introduced Central } \\
\text { America and }\end{array}$ & $\begin{array}{l}0.00 / \\
0.00\end{array}$ & $\begin{array}{l}0.13 / \\
0.00\end{array}$ & leaves (B) & $\begin{array}{l}\text { against bleedings (C) } \\
\text { against uterus complaints (C) }\end{array}$ & $\begin{array}{l}\text { fresh leaves directly on the wound/ topical } \\
\text { (C) }\end{array}$ \\
\hline Asteraceae & $\begin{array}{l}\text { between bushes } \\
\text { on open }\end{array}$ & $\begin{array}{l}\text { naturalized in } \\
\text { Colombia }^{3}\end{array}$ & & & & & infusion/ oral (C) \\
\hline ALCG 139 & pastures & & & & & pains in muscles (Z) & maceration/ topical-emplast (Z) \\
\hline
\end{tabular}

Santa María 
Table 4 List of medicinal plant species reported in Zetaquira and Campo Hermoso including ethnobotanical characteristics (Continued)

\begin{tabular}{|c|c|c|c|c|c|c|c|}
\hline $\begin{array}{l}\text { Conyza filaginoides (DC.) } \\
\text { Hieron } \\
\text { Asteraceae } \\
\text { ALCG } 110 \\
\text { Venadillo }\end{array}$ & $\begin{array}{l}\text { herb } \\
\text { spontaneous in } \\
\text { solar with some } \\
\text { cultivated trees }\end{array}$ & $\begin{array}{l}\text { native Central } \\
\text { Colombian Andeans } \\
3\end{array}$ & $\begin{array}{l}0.04 / \\
0.00\end{array}$ & $\begin{array}{l}0.00 / \\
0.00\end{array}$ & leaves and stems (Z) & skin infections and rash (Z) & $\begin{array}{l}\text { fresh extract by maceration/topical-massage } \\
\text { (Z) }\end{array}$ \\
\hline $\begin{array}{l}\text { Pyrethrum parthenium (L.) } \\
\text { Sw. }\end{array}$ & \multirow[t]{3}{*}{$\begin{array}{l}\text { herb cultivated } \\
\text { in small garden }\end{array}$} & \multirow[t]{3}{*}{$\begin{array}{l}\text { introduced Eurasian, } \\
\text { meridional-boreal }^{3}\end{array}$} & \multirow[t]{3}{*}{$\begin{array}{l}0.36 / \\
0.28\end{array}$} & \multirow[t]{3}{*}{$\begin{array}{l}0.00 / \\
0.00\end{array}$} & \multirow[t]{2}{*}{$\begin{array}{l}\text { leaves }{ }^{\circ} 2^{*} 2 \text { and stems } \\
(\mathrm{Z})^{*} 2\end{array}$} & $\begin{array}{l}\text { intestinal complaints (Z) } \\
\text { relaxant (Z) }\end{array}$ & \multirow[t]{3}{*}{$\begin{array}{l}\text { infusion/ oral (Z) } \\
\text { fresh maceration/ emplast (Z) }\end{array}$} \\
\hline Asteraceae & & & & & & & \\
\hline $\begin{array}{l}\text { ALCG } 75 \\
\text { Manzanilla } \\
\text { Feverfew } \\
\text { Wild chamomille }\end{array}$ & & & & & flowers (Z) & uterus cramps (Z) & \\
\hline $\begin{array}{l}\text { Galinsoga parviflora Cav. } \\
\text { Asteraceae } \\
\text { ALCG } 90 \\
\text { Guacas }\end{array}$ & $\begin{array}{l}\text { herb cultivated } \\
\text { in homegarden }\end{array}$ & $\begin{array}{l}\text { native Colombia, } \\
\text { South America }{ }^{3,8}\end{array}$ & $\begin{array}{l}0.08 / \\
0.04\end{array}$ & $\begin{array}{l}0.19 / \\
0.06\end{array}$ & leaves and stems (C) & $\begin{array}{l}\text { againts excess of stomach acids (B) } \\
\text { condiment herb (B) } \\
\text { against white plaques in mouth (C) } \\
\text { intestinal parasits (C) }\end{array}$ & $\begin{array}{l}\text { decoction/ oral (B) } \\
\text { decoction/ oral (B) } \\
\text { fresh extract by maceration in water/ oral (C) }\end{array}$ \\
\hline$\underset{\circ}{\text { Matricaria chamomilla L. * }}$ & \multirow[t]{3}{*}{$\begin{array}{l}\text { herb cultivated } \\
\text { in homegarden }\end{array}$} & \multirow[t]{3}{*}{$\begin{array}{l}\text { introduced Iran and } \\
\text { Europe }\end{array}$} & \multirow[t]{3}{*}{$\begin{array}{l}0.32 / \\
0.32\end{array}$} & \multirow[t]{3}{*}{$\begin{array}{l}0.38 / \\
0.38\end{array}$} & \multirow[t]{2}{*}{ leaves and flowers (B) } & $\begin{array}{l}\text { stomach complaints (B) } \\
\text { relaxant (B) }\end{array}$ & $\begin{array}{l}\text { decoction or infusion (B) } \\
\text { infusion/ oral (B) }\end{array}$ \\
\hline Asteraceae & & & & & & rheumatism (Z) & $\begin{array}{l}\text { fresh extract by maceration/ topical-massage } \\
\text { (Z) }\end{array}$ \\
\hline $\begin{array}{l}\text { ALCG } 96 \\
\text { Manzanilla dulce } \\
\text { Roman chamomile, } \\
\text { German chamomile }\end{array}$ & & & & & $\begin{array}{l}\text { leaves, stems and } \\
\text { flowers (B) }\end{array}$ & $\begin{array}{l}\text { colds, spams and cramps in muscles } \\
\text { (C) }\end{array}$ & $\begin{array}{l}\text { decoction/ oral and fresh extract by } \\
\text { maceration/ topical-massage }(C)\end{array}$ \\
\hline $\begin{array}{l}\text { Taraxacum officinales F.H. } \\
\text { Wigg. } \\
\text { * }\end{array}$ & \multirow{5}{*}{$\begin{array}{l}\text { herb } \\
\text { spontaneous in } \\
\text { a farm with a } \\
\text { large patch of } \\
\text { secondary forest }\end{array}$} & \multirow[t]{5}{*}{ introduced Europe ${ }^{3}$} & \multirow[t]{5}{*}{$\begin{array}{l}0.16 / \\
0.12\end{array}$} & \multirow[t]{5}{*}{$\begin{array}{l}0.25 / \\
0.06\end{array}$} & \multirow[t]{5}{*}{$\begin{array}{l}\text { leaves (B) } \\
* 2\end{array}$} & cardiovascular complaints (B) & $\begin{array}{l}\text { infusion/ oral (B) } \\
\text { infusion/ oral (C) }\end{array}$ \\
\hline Asteraceae & & & & & & enhance lungs function (Z) & infusion/ oral (Z) \\
\hline ALCG 104 & & & & & & rheumatism (Z) & decoction/ oral (Z) \\
\hline Diente de león & & & & & & liver cleanse (Z) & maceration/ topical-emplast (Z) \\
\hline Dandelion & & & & & & & infusion/ oral (Z) \\
\hline $\begin{array}{l}\text { Artemisia absinthium L. }{ }^{*} \\
(\mathrm{e}-\mathrm{CH})\end{array}$ & $\begin{array}{l}\text { herb cultivated } \\
\text { in homegarden }\end{array}$ & $\begin{array}{l}\text { introduced Europe } \\
\text { and West Asia }\end{array}$ & $\begin{array}{l}0.32 / \\
0.16\end{array}$ & $\begin{array}{l}0.19 / \\
0.13\end{array}$ & $\begin{array}{l}\text { leaves (B) } \\
\text { leaves (Z) }\end{array}$ & $\begin{array}{l}\text { stomach ache (B) } \\
\text { liver cleanser (C) }\end{array}$ & infusion/ oral (B) \\
\hline
\end{tabular}


Table 4 List of medicinal plant species reported in Zetaquira and Campo Hermoso including ethnobotanical characteristics (Continued)

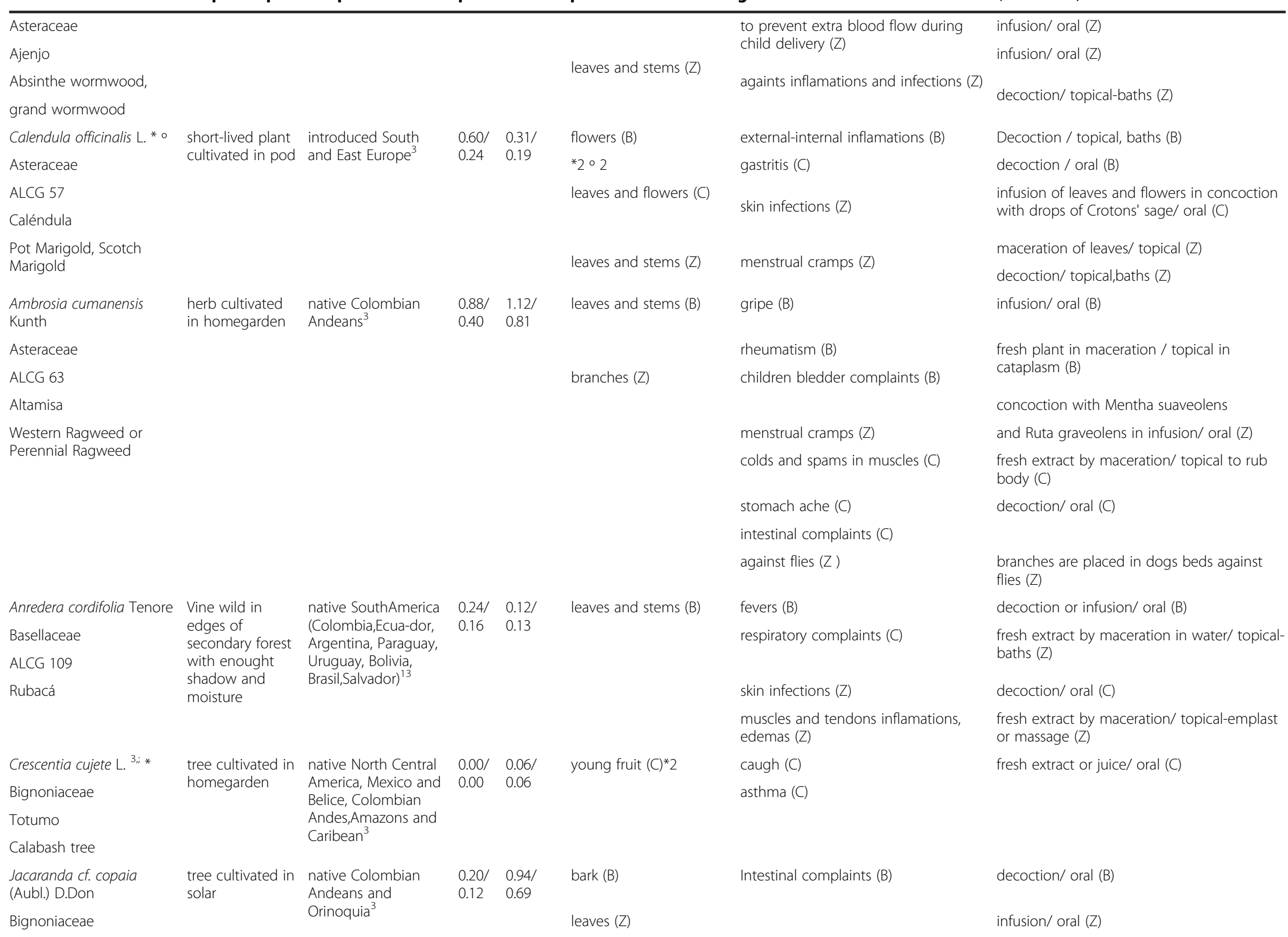


Table 4 List of medicinal plant species reported in Zetaquira and Campo Hermoso including ethnobotanical characteristics (Continued)

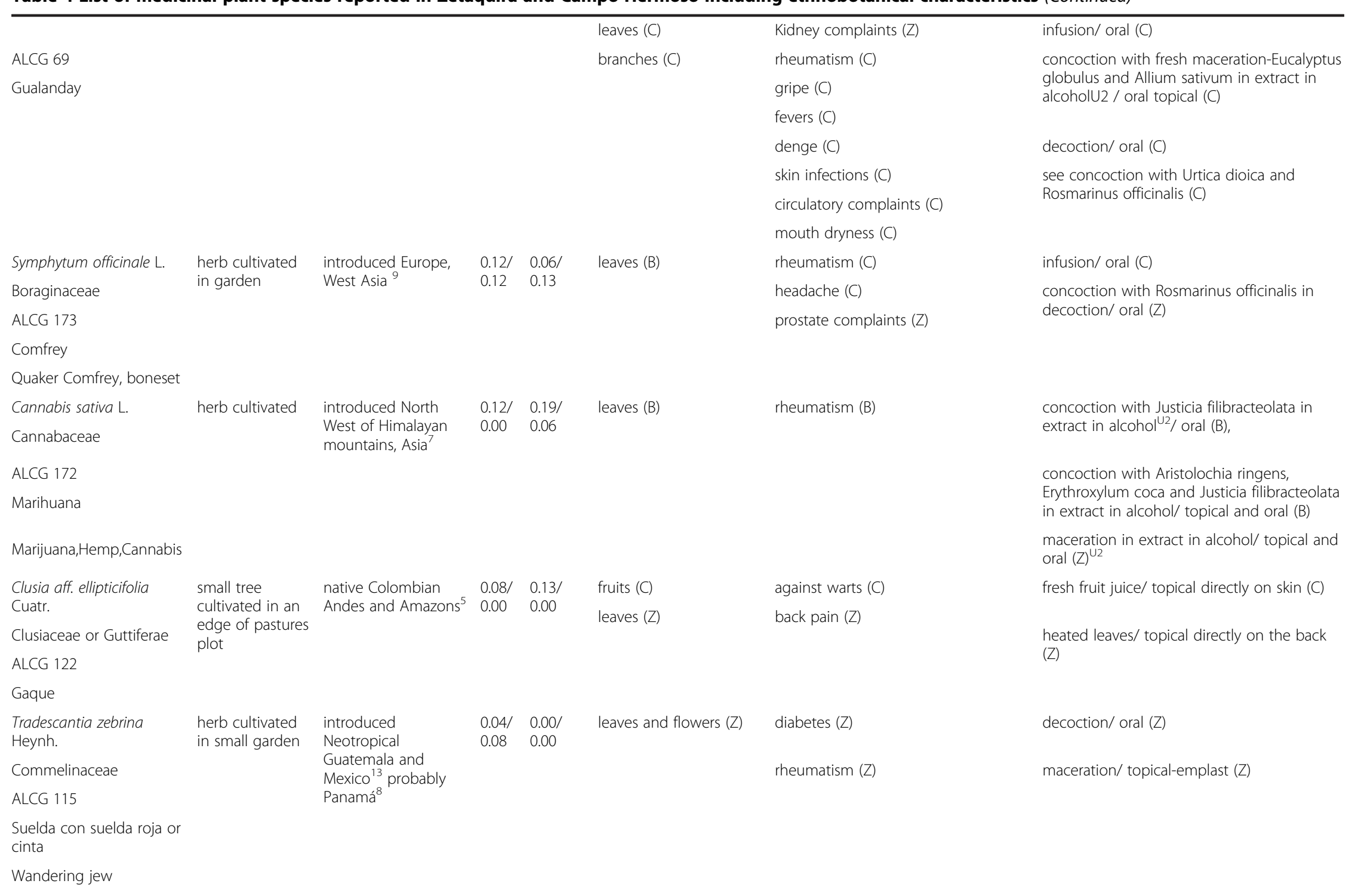


Table 4 List of medicinal plant species reported in Zetaquira and Campo Hermoso including ethnobotanical characteristics (Continued)

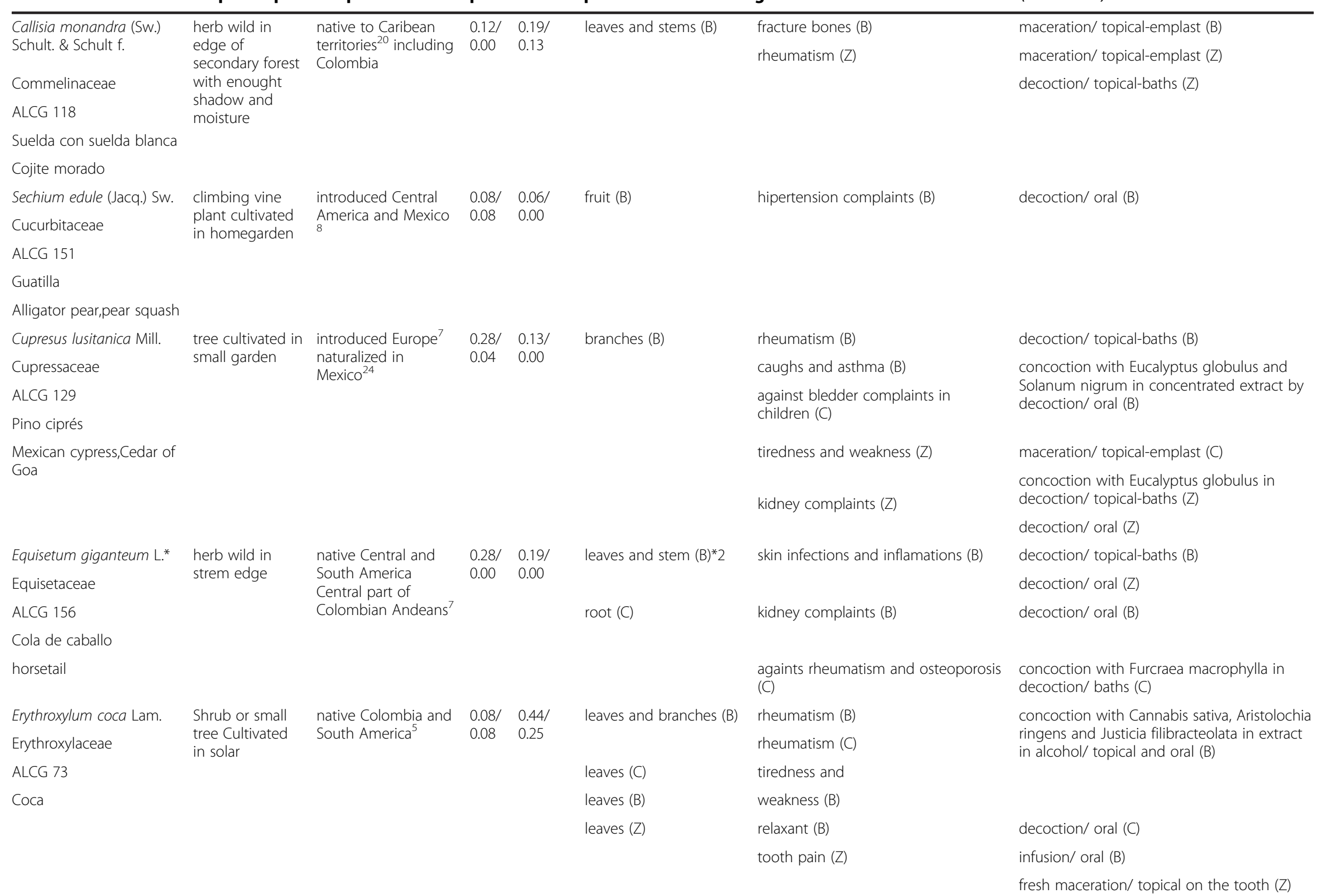


Table 4 List of medicinal plant species reported in Zetaquira and Campo Hermoso including ethnobotanical characteristics (Continued)

\begin{tabular}{|c|c|c|c|c|c|c|c|}
\hline $\begin{array}{l}\text { Croton Funckianus Muell. } \\
\text { Arg. }\end{array}$ & $\begin{array}{l}\text { tree wild in } \\
\text { edge of route }\end{array}$ & $\begin{array}{l}\text { native Colombian } \\
\text { Andes }^{5}\end{array}$ & $\begin{array}{l}0.00 / \\
0.00\end{array}$ & $\begin{array}{l}0.06 / \\
0.06\end{array}$ & leaves $(C)$ & tooth pain $(C)$ & maceration/ topical-emplast (B) \\
\hline \multicolumn{8}{|l|}{ Euphorbiaceae } \\
\hline \multicolumn{8}{|l|}{ ALCG 120} \\
\hline \multicolumn{8}{|l|}{ Sangregado } \\
\hline Mimosa pudica L. & herb wild in & native South and & $0.12 /$ & $0.56 /$ & leaves (B) & tooth pain (B) & fresh maceration/ topical-emplast (B) \\
\hline Fabaceae & open pastures & Central America & & & & rheumatism (C) & decoction/ topical-bath (C) \\
\hline ALCG 175 & & & & & & for helping children to fall asleep (C) & \\
\hline \multicolumn{8}{|l|}{ Dormidera } \\
\hline \multicolumn{8}{|l|}{ Sensitive Plant, Mimosa } \\
\hline $\begin{array}{l}\text { Senna obtusifolia (L.) Irwin } \\
\text { \& Barnaby }\end{array}$ & $\begin{array}{l}\text { shrub } \\
\text { spontaneous in } \\
\text { a sideroad }\end{array}$ & $\begin{array}{l}\text { introduced } 48 \text { lower } \\
\text { states, Virgin Islands, } \\
\text { Puerto Rico }\end{array}$ & $\begin{array}{l}0.12 / \\
0.08\end{array}$ & $\begin{array}{l}0.50 / \\
0.06\end{array}$ & branches (B) & fever (B) & \multirow{2}{*}{$\begin{array}{l}\text { decoction/ topical-bath (C) } \\
\text { concoction with Trichanthera gigantea and } \\
\text { Cestrum mariquitense in decoction/ topical- } \\
\text { bath }(\mathrm{Z})\end{array}$} \\
\hline Fabaceae & & & & & leaves $(C)$ & gripe (C) & \\
\hline ALCG 161 & & & & & branches (C) & headache (C) & decoction/ oral (C) \\
\hline Alcaparro & & & & & & $\begin{array}{l}\text { for healing uterus after giving birth } \\
\text { (C) }\end{array}$ & fresh extract by \\
\hline Sicklepod & & & & & branches (B) & $\begin{array}{l}\text { to stop noise bleeding in children } \\
\text { (Z) }\end{array}$ & $\begin{array}{l}\text { maceration/ topical-massage or bath (C) } \\
\text { decoction/ topical-bath (C) } \\
\text { decoction in milk/ topical-bath (Z) }\end{array}$ \\
\hline Juglans neotropica Diels & \multirow{6}{*}{$\begin{array}{l}\text { tree cultivated in } \\
\text { solar within a } \\
\text { secondary } \\
\text { forests well } \\
\text { drened }\end{array}$} & \multirow{6}{*}{$\begin{array}{l}\text { native Amazons, } \\
\text { Orinoquia Central } \\
\text { Andes Range }^{7}\end{array}$} & \multirow{6}{*}{$\begin{array}{l}0.40 / \\
0.20\end{array}$} & \multirow{6}{*}{$\begin{array}{l}0.00 / \\
0.00\end{array}$} & leaves (Z) & allergies (Z) & \multirow{2}{*}{$\begin{array}{l}\text { infusion/ oral (Z) } \\
\text { decoction/ topical in baths (Z) }\end{array}$} \\
\hline$(\mathrm{e}-\mathrm{CH} / \mathrm{Z})$ & & & & & leaves and branches (Z) & $\begin{array}{l}\text { diaper rash and other skin complaints } \\
\text { as acne and feets' fungus (Z) }\end{array}$ & \\
\hline Juglandaceae & & & & & & & decoction/ topical in hip baths (Z) \\
\hline \multicolumn{4}{|l|}{ ALCG 67} & & & & \\
\hline \multicolumn{4}{|c|}{ uterus cleaning (Z) } & & & & \\
\hline \multicolumn{4}{|l|}{ Colombian walnut } & & & & \\
\hline Melissa officinalis L.* $\circ$ & \multirow{5}{*}{$\begin{array}{l}\text { herb cultivated } \\
\text { in homegarden }\end{array}$} & \multirow{5}{*}{$\begin{array}{l}\text { introduced Europe } \\
\text { and Asia }\end{array}$} & \multirow{5}{*}{$\begin{array}{l}0.88 / \\
0.72\end{array}$} & \multirow{5}{*}{$\begin{array}{l}0.75 / \\
0.62\end{array}$} & \multirow[t]{5}{*}{ leaves ${ }^{\circ} 2$ and stems $(B)^{*} 2$} & gripe (B) & \multirow{5}{*}{$\begin{array}{l}\text { decoction/ oral (B) or fresh extract by } \\
\text { maceration in water/ oral (B) or infusion/ oral } \\
\text { (B) }\end{array}$} \\
\hline Lamiaceae & & & & & & fevers (B) & \\
\hline ALCG 92 & & & & & & relaxant (B) & \\
\hline Toronjil & & & & & & stomach ache (B) & \\
\hline Common balm & & & & & & intestine complaints (B) & \\
\hline $\begin{array}{l}\text { Ocimum campechianum } \\
\text { Mill. }\end{array}$ & $\begin{array}{l}\text { herb cultivated } \\
\text { in homegarden }\end{array}$ & $\begin{array}{l}\text { native North, Central } \\
\text { and Northern South } \\
\text { America }\end{array}$ & $\begin{array}{l}0.28 / \\
0.16\end{array}$ & $\begin{array}{l}0.63 / \\
0.31\end{array}$ & leaves (B) & Intestinal complaints (B) & infusion/ oral (B) \\
\hline
\end{tabular}


Table 4 List of medicinal plant species reported in Zetaquira and Campo Hermoso including ethnobotanical characteristics (Continued)

\begin{tabular}{|c|c|c|c|c|c|c|c|}
\hline Lamiaceae & & & & & \multirow[t]{2}{*}{ seeds (B) } & eye dust (B) & one seed is placed in the eye/ topical (B) \\
\hline ALCG 74 & & & & & & gripe (Z) & infusion/ oral (Z) \\
\hline Albahaca & & & & & leaves (Z) & & \\
\hline \multicolumn{8}{|l|}{ Basil, Wild mosquito plant } \\
\hline Mentha viridis $L$. & \multirow{4}{*}{$\begin{array}{l}\text { herb cultivated } \\
\text { in homegarden }\end{array}$} & \multirow[t]{4}{*}{ introduced Europe ${ }^{3}$} & \multirow{4}{*}{$\begin{array}{l}0.36 / \\
0.20\end{array}$} & \multirow{4}{*}{$\begin{array}{l}0.50 / \\
0.38\end{array}$} & \multirow[t]{4}{*}{ leaves (B) } & gripe (B) & infusion/ oral (B) \\
\hline Lamiaceae & & & & & & stomach ache (B) & infusion or decoction/ oral (B) \\
\hline ALCG 91 Menta & & & & & & inflammations of intestine (B) & fresh extract by maceration in water/ oral (C) \\
\hline Spearmint & & & & & & & infusion/ oral (B) \\
\hline $\begin{array}{l}\text { Satureja brownei (Sw.) } \\
\text { Briq. }\end{array}$ & \multirow[t]{4}{*}{$\begin{array}{l}\text { herb cultivated } \\
\text { in small garden }\end{array}$} & \multirow{4}{*}{$\begin{array}{l}\text { native South } \\
\text { America, Colombian } \\
\text { Andean Ranges }^{3}\end{array}$} & \multirow[t]{4}{*}{$\begin{array}{l}0.60 / \\
0.20\end{array}$} & \multirow[t]{4}{*}{$\begin{array}{l}0.19 / \\
0.06\end{array}$} & \multirow[t]{4}{*}{ leaves and stems (B) } & $\begin{array}{l}\text { against bledder complaints in } \\
\text { children (B) }\end{array}$ & maceration/ topical-emplast (B) \\
\hline Lamiaceae & & & & & & condiment herb (B) & combination with food/ oral (B) \\
\hline ALCG 130 & & & & & & stomach ache (Z) & infusion/ oral (Z) \\
\hline Poleo & & & & & & enhance blood cleaning (Z) & infusion/ oral (Z) \\
\hline Origanum majorana L.* & \multirow{5}{*}{$\begin{array}{l}\text { herb cultivated } \\
\text { in solar }\end{array}$} & \multirow{5}{*}{$\begin{array}{l}\text { introduced Asia } \\
\text { menor }^{13}\end{array}$} & \multirow{5}{*}{$\begin{array}{l}0.36 / \\
0.28\end{array}$} & \multirow{5}{*}{$\begin{array}{l}0.06 / \\
0.00\end{array}$} & \multirow[t]{5}{*}{ leaves $(Z) * 2$} & stomach aches and & infusion or decoction/ \\
\hline Lamiaceae & & & & & & & \\
\hline ALCG 76 & & & & & & intestine complaints (Z) & oral (Z) \\
\hline Mejorana & & & & & & prostata complaints (Z) & infusion/ oral (Z) \\
\hline $\begin{array}{l}\text { knotted marjoram,sweet } \\
\text { marjoram }\end{array}$ & & & & & & kidney complaints (Z) & infusion/ oral (Z) \\
\hline \multirow[t]{2}{*}{ Rosmarinus officinalis $L . * 0$} & \multirow{8}{*}{$\begin{array}{l}\text { woody herb } \\
\text { cultivated in } \\
\text { homegarden }\end{array}$} & \multirow{8}{*}{$\begin{array}{l}\text { introduced Península } \\
\text { Ibérica, Western } \\
\text { Mediterranean }\end{array}$} & \multirow{8}{*}{$\begin{array}{l}0.44 / \\
0.44\end{array}$} & \multirow{8}{*}{$\begin{array}{l}0.31 / \\
0.19\end{array}$} & leaves (B) & lungs complaints (B) & infusion/ oral (C) \\
\hline & & & & & $* 2 \circ 2$ & tooth pain (B) & decoction in milk/ oral (Z) \\
\hline$(\mathrm{e}-\mathrm{Z})$ & & & & & & hipertension complaints (B) & maceration/ topical-emplast on tooth (B) \\
\hline Lamiaceae & & & & & & against hair loss (B) & infusion/ oral (B) \\
\hline ALCG 149 & & & & & & muscles pain (C) & $\begin{array}{l}\text { fresh extract by maceration/ topical-massage } \\
\text { (C) }\end{array}$ \\
\hline Romero & & & & & leaves and stems (B) & rheumatism (C) & decoction/ topical-baths (Z) \\
\hline \multirow[t]{2}{*}{ Rosemary } & & & & & & stomach ache (Z) & $\begin{array}{l}\text { fresh extract by maceration/topical-massage } \\
\text { (C) }\end{array}$ \\
\hline & & & & & & tiredness (Z) & infusion/ oral (Z) \\
\hline Mentha suaveolens Ehrh. & \multirow{5}{*}{$\begin{array}{l}\text { herb cultivated } \\
\text { in homegarden }\end{array}$} & \multirow[t]{5}{*}{ introduced Europe ${ }^{3}$} & \multirow{5}{*}{$\begin{array}{l}1.20 / \\
1.04\end{array}$} & \multirow{5}{*}{$\begin{array}{l}0.94 / \\
0.94\end{array}$} & leaves (B) & stomach ache (B) & infusion or decoction/ oral (B) \\
\hline Lamiaceae & & & & & shoots (Z) & & fresh extract by maceration/ oral (B) \\
\hline ALCG 127 & & & & & leaves and stems (Z) & & $\begin{array}{l}\text { infusion or fresh extract by maceration/ oral } \\
\text { (B) }\end{array}$ \\
\hline Yerbabuena blanca & & & & & leaves $(Z)$ & headaches (B) & $\begin{array}{l}\text { concoction with Allium sativum cloves, in } \\
\text { concentrated extract by decoction/ oral (Z) }\end{array}$ \\
\hline Round leaved mint & & & & & leaves and stems (Z) & intestinal inflamations (B) & \\
\hline
\end{tabular}




\begin{tabular}{|c|c|c|c|c|c|c|c|}
\hline & & & & & & intestinal parasits (Z) & \\
\hline & & & & & & against infections and inflamations (Z) & $\begin{array}{l}\text { Irichanthera gigantea and Phytolacca } \\
\text { rivinoidesin decoction/ topical-baths (Z) }\end{array}$ \\
\hline & & & & & & fevers (Z) & infusion/ oral (Z) \\
\hline & & & & & & menstrual cramps (Z) & $\begin{array}{l}\text { concoction with Ambrosia cumanensis and } \\
\text { Ruta graveolens in decoction/ oral (Z) }\end{array}$ \\
\hline & & & & & & diaper rash (Z) & decoction/ topical-baths (Z) \\
\hline \multirow[t]{2}{*}{ Persea americana Mill. } & \multirow{6}{*}{$\begin{array}{l}\text { tree cultivated in } \\
\text { solar }\end{array}$} & \multirow{6}{*}{$\begin{array}{l}\text { native Central and } \\
\text { South America }{ }^{7}\end{array}$} & \multirow{6}{*}{$\begin{array}{l}0.04 / \\
0.04\end{array}$} & \multirow{6}{*}{$\begin{array}{l}0.25 / \\
0.13\end{array}$} & \multirow[t]{2}{*}{ shoots $(C)$} & gripe $(C)$ & \multirow{2}{*}{$\begin{array}{l}\text { decoction and concoction with fresh extract } \\
\text { of Verbena littoralis and Citrus limon juice/ } \\
\text { oral (C) }\end{array}$} \\
\hline & & & & & & apendicitis (C) & \\
\hline Lauraceae & & & & & shoots (Z) & sensitive gums (Z) & decoction/ oral (C) \\
\hline ALCG 100 & & & & & fruit $(C)$ & against dry hair (C) & maceration/ topical (Z) \\
\hline Aguacate & & & & & fruit (Z) & regulator of digestion (Z) & maceration/ topical-massage (C) \\
\hline Avocado, butter pear & & & & & & & combination with food/ oral (Z) \\
\hline \multirow[t]{2}{*}{ Allium fistulosum L. } & \multirow{8}{*}{$\begin{array}{l}\text { gregarious herb } \\
\text { cultivated in } \\
\text { homegarden }\end{array}$} & \multirow{8}{*}{$\begin{array}{l}\text { introduced Siberia ; } \\
\text { Altai montains in } \\
\text { Siberia }^{7}\end{array}$} & \multirow{8}{*}{$\begin{array}{l}0.12 / \\
0.08\end{array}$} & \multirow{8}{*}{$\begin{array}{l}0.31 / \\
0.19\end{array}$} & roots (B) & intestinal obstipation (B) & decoction/ oral (B) \\
\hline & & & & & & & fresh extract by maceration/ oral (C) \\
\hline Liliaceae & & & & & leaves (B) & stomach ache (B) & decoction/ oral (Z) \\
\hline Cebolla larga & & & & & leaves (B) & condiment herb (B) & combination with food (B) \\
\hline \multirow[t]{4}{*}{ Scallion, green onion } & & & & & young leaf $(C)$ & $\begin{array}{l}\text { against intestinal obstipation in } \\
\text { newborns }(C)\end{array}$ & fresh leaf as suppository/ rectal (C) \\
\hline & & & & & roots $(C)$ & lost of memory (C) & \\
\hline & & & & & leaves (B) & fever $(Z)$ & decoction/ oral (C) \\
\hline & & & & & & & $\begin{array}{l}\text { maceration/ topical-emplast on head-front } \\
\text { (Z) }\end{array}$ \\
\hline Allium sativum $\mathrm{L} .{ }^{*} \circ$ & \multirow[t]{5}{*}{$\begin{array}{l}\text { herb cultivated } \\
\text { in pod }\end{array}$} & \multirow{5}{*}{$\begin{array}{l}\text { introduced } \\
\text { Southwestern Asia; } \\
\text { Central Asia }{ }^{7}\end{array}$} & \multirow[t]{5}{*}{$\begin{array}{l}0.32 / \\
0.04\end{array}$} & \multirow[t]{5}{*}{$\begin{array}{l}0.12 / \\
0.06\end{array}$} & bulbus $(B) * 2 \circ 2$ & gripe $(C)$ & $\begin{array}{l}\text { concoction with alcohol-Aguardiente } \mathrm{U} 2 / \text { oral } \\
\text { (C) }\end{array}$ \\
\hline Liliaceae & & & & & & fevers $(C)$ & decoction/ oral (C) \\
\hline Ajo & & & & & & againts intestinal worms $(C)$ & $\begin{array}{l}\text { concoction with alcohol-Aguardiente- }{ }^{\mathrm{U} 2} / \text { oral } \\
\text { (Z) }\end{array}$ \\
\hline \multirow[t]{2}{*}{ Garlic } & & & & & & againts amebiasis (Z) & $\begin{array}{l}\text { concoction with shoots of Menta suaveolens } \\
\text { in concentrated extract by decoction/ oral } \\
\text { (Z) }\end{array}$ \\
\hline & & & & & & rheumatism (Z) & $\begin{array}{l}\text { concoction with Justicia filibracteolata and } \\
\text { Aristolochia ringens in extract in alcohol }{ }^{\mathrm{U}} \text { - } \\
\text { (Z) }\end{array}$ \\
\hline Althaea officinalis L. ${ }^{\circ}$ & \multirow{2}{*}{$\begin{array}{l}\text { herb cultivated } \\
\text { in pod }\end{array}$} & \multirow{2}{*}{$\begin{array}{l}\text { introduced } \\
\text { Mediterranean }\end{array}$} & \multirow{2}{*}{$\begin{array}{l}0.08 / \\
0.04\end{array}$} & \multirow{2}{*}{$\begin{array}{l}0.38 / \\
0.00\end{array}$} & leaves (C) & kidney complaints (C) & infusion/ oral (C) \\
\hline Malvaceae & & & & & flowers (C) & caugh $(C)$ & fresh extract by maceration/ oral (Z) \\
\hline
\end{tabular}


Table 4 List of medicinal plant species reported in Zetaquira and Campo Hermoso including ethnobotanical characteristics (Continued)

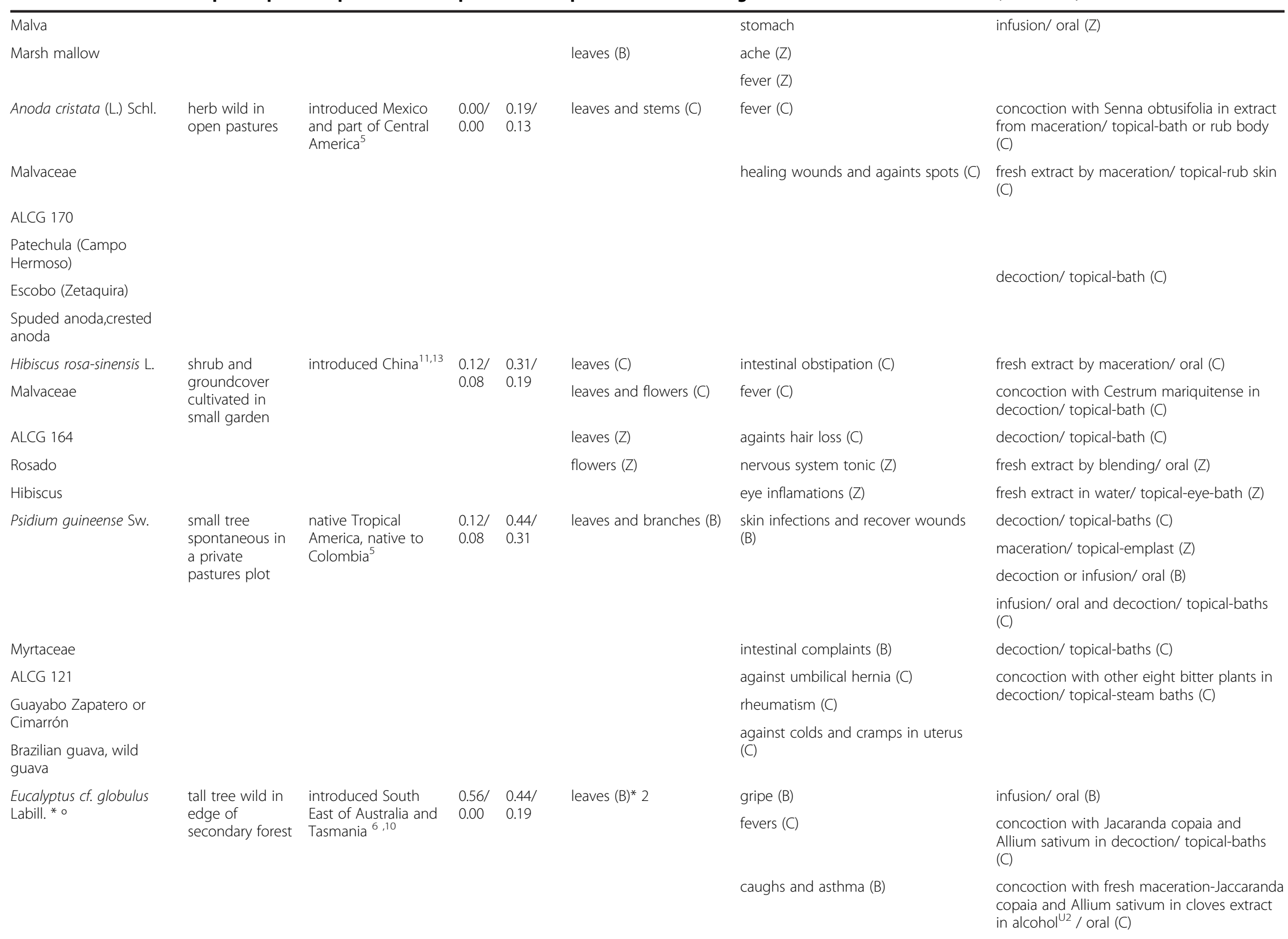


Table 4 List of medicinal plant species reported in Zetaquira and Campo Hermoso including ethnobotanical characteristics (Continued)

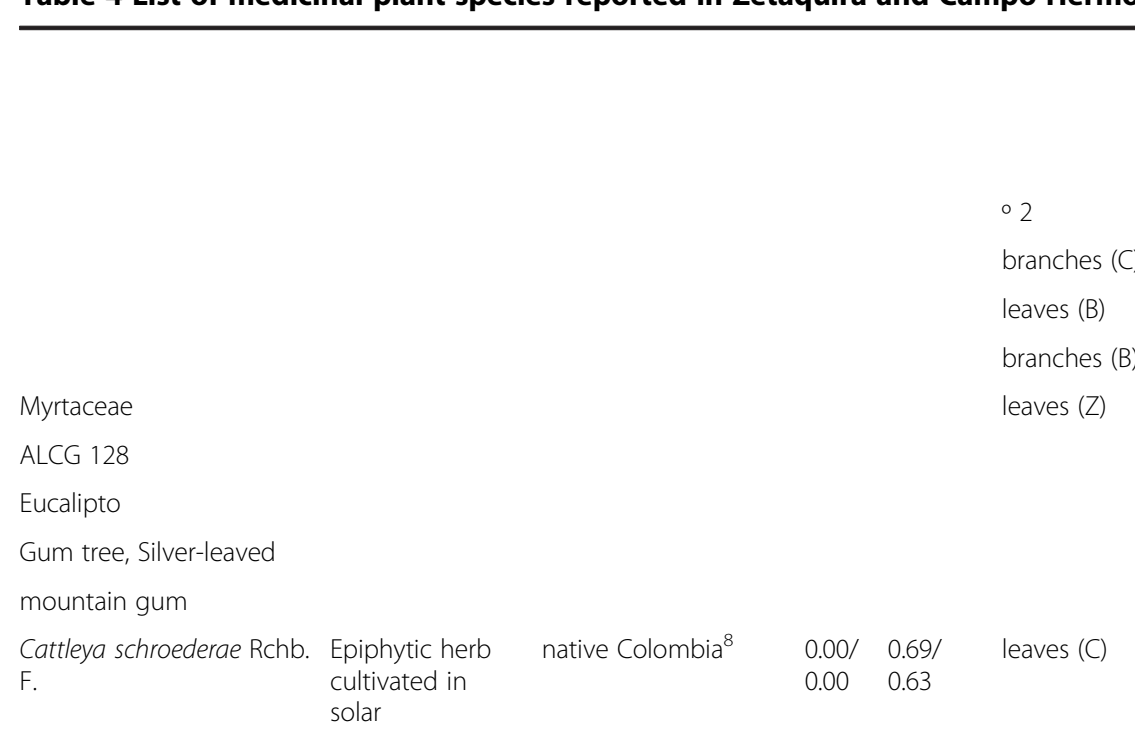

\section{Orchidaceae}

ALCG 165

Lirio

Easter orchid, Baron

schroeder's

cattleya

Petiveria alliacea L. *

$$
\begin{array}{cl}
\text { herb cultivated } & \text { native Andean } \\
\text { in small garden } & \text { (specially Peruvian } \\
& \text { Amazonas), Caribean } \\
& \text { and South east of } \\
& \text { Colombia }
\end{array}
$$

concoction with Solanum nigrum and

Cupresus lusitanica in concentrated extract

by decoction/ oral (Z)

decoction/ topical-baths (B)

concoction with Cupresus lusitanica in decoction/ topical-baths (Z) typhus (C)

denge (C)

fever (C)

intestinal

complaints (C)
Phytolaccaceae

ALCG 136

Anamú

Anamú plant fresh extract by maceration of one leafe/ oral (C)

extract by blending in concoction with water/ oral (C)

fresh extract by maceration of a piece of leafe in concoction with water/ oral (C)

infusion/ oral (C)

decoction/ oral (C)

extract by maceration/ topical-rinsing mouth out/ (C)

against lacerations in mouth (C)

headache (C) infusion/ oral (C) 
Table 4 List of medicinal plant species reported in Zetaquira and Campo Hermoso including ethnobotanical characteristics (Continued)

\begin{tabular}{|c|c|c|c|c|c|c|c|}
\hline \multirow{3}{*}{$\begin{array}{l}\text { Phytolacca rivinoides } \\
\text { Kunth \& C.D.Bouché }\end{array}$} & \multirow{4}{*}{$\begin{array}{l}\text { shrub } \\
\text { spontaneous in } \\
\text { a solar }\end{array}$} & \multirow{4}{*}{$\begin{array}{l}\text { native Central and } \\
\text { South America, } \\
\text { Colombian Andeans }{ }^{7}\end{array}$} & \multirow{5}{*}{$\begin{array}{l}0.08 / \\
0.00\end{array}$} & \multirow{3}{*}{$\begin{array}{l}0.38 / \\
0.25\end{array}$} & \multirow[t]{3}{*}{ leaves and branches (B) } & rheumatism (C) & \multirow{4}{*}{$\begin{array}{l}\text { concoction with Mentha suaveolens,Piper } \\
\text { bogotense and Trichanthera giganteain } \\
\text { decoction/ topical-baths (Z) }\end{array}$} \\
\hline & & & & & & erisipela (C) & \\
\hline & & & & & & inflamations and skin infections (C) & \\
\hline Phytolaccaceae & & & & & & inflamations and skin infections (Z) & \\
\hline \multicolumn{7}{|l|}{ ALCG 146} & \\
\hline \multicolumn{8}{|l|}{ Guaba, Cargamanto } \\
\hline \multirow[t]{2}{*}{ Piper hispidum Sw. } & \multirow{6}{*}{$\begin{array}{l}\text { shrub cultivated } \\
\text { in solar/it can } \\
\text { also occur in } \\
\text { wild }\end{array}$} & \multirow{6}{*}{$\begin{array}{l}\text { native Central and } \\
\text { South America and } \\
\text { native to Caribean } \\
\text { territories }{ }^{20}\end{array}$} & \multirow{6}{*}{$\begin{array}{l}0.00 / \\
0.00\end{array}$} & \multirow{6}{*}{$\begin{array}{l}0.44 / \\
0.38\end{array}$} & \multirow[t]{6}{*}{ leaves and stems (C) } & high blood presure (C) & \multirow{4}{*}{$\begin{array}{l}\text { fresh extract by maceration in water/ oral (C) } \\
\text { concoction with Verbena littoralis in } \\
\text { decoction/ topical-bath (C) } \\
\text { decoction / topical-bath (C),steam baths/ } \\
\text { topical(C) }\end{array}$} \\
\hline & & & & & & fevers (C) & \\
\hline Piperaceae & & & & & & tiredness and weakness (C) & \\
\hline ALCG 85 & & & & & & rheumatism (C) & \\
\hline \multicolumn{3}{|l|}{ Cordoncillo negro } & & & & & \\
\hline \multicolumn{3}{|l|}{ Jamaican pepper } & & & & & \\
\hline \multirow[t]{3}{*}{ Piper cf. bogotense C.DC. } & \multirow{6}{*}{$\begin{array}{l}\text { shrub cultivated } \\
\text { in solar/it can } \\
\text { also occur in } \\
\text { wild }\end{array}$} & \multirow{6}{*}{$\begin{array}{l}\text { native North of } \\
\text { South America }{ }^{12}\end{array}$} & \multirow{6}{*}{$\begin{array}{l}0.08 / \\
0.00\end{array}$} & \multirow{6}{*}{$\begin{array}{l}0.50 / \\
0.25\end{array}$} & \multirow[t]{2}{*}{ leaves $(C)$} & high blood presure (C) & \multirow{6}{*}{$\begin{array}{l}\text { fresh extract by maceration in water/ oral (C) } \\
\text { extract in water/ oral (C) } \\
\text { concoction with Mentha suaveolens and } \\
\text { Phytolacca rivinoides in decoction/ topical- } \\
\text { bath (Z) }\end{array}$} \\
\hline & & & & & & fevers (C) & \\
\hline & & & & & leaves and stems ( $Z$ ) & mouth dryness $(C)$ & \\
\hline Piperaceae & & & & & & skin infections and inflamations (Z) & \\
\hline \multicolumn{3}{|l|}{$\begin{array}{l}\text { ALCG } 81 \\
\text { Cordoncillo blanco }\end{array}$} & & & & & \\
\hline \multicolumn{3}{|l|}{ Cordoncillo blanco } & & & & & \\
\hline \multirow[t]{2}{*}{ Plantago major L.* } & \multirow{11}{*}{$\begin{array}{l}\text { herb } \\
\text { spontaneous in } \\
\text { homegarden }\end{array}$} & \multirow{11}{*}{$\begin{array}{l}\text { introduced Europe } \\
\text { and Asia }\end{array}$} & \multirow{11}{*}{$\begin{array}{l}0.32 / \\
0.16\end{array}$} & $0.81 /$ & leaves $(B)^{*} 2$ & eye dust and infections (B) & decoction/ topical eye bath (C) \\
\hline & & & & 0.31 & & & $\begin{array}{l}\text { fresh extract by destilation in glass bottle/ } \\
\text { topical-eye drops ( } Z \text { ) }\end{array}$ \\
\hline Plantaginaceae & & & & & & gastritis (B) & fresh extract by maceration/ oral (B) \\
\hline & & & & & & & decoction/ oral (C) \\
\hline & & & & & & & fresh extract by maceration/ oral (Z) \\
\hline & & & & & & & decoction/ oral (C) \\
\hline & & & & & & & decoction/ oral (C) \\
\hline & & & & & & & $\begin{array}{l}\text { fresh maceration in concoction with honey/ } \\
\text { topical-emplast (C) }\end{array}$ \\
\hline & & & & & & liver cleanser (B) & concoction with Calendula officinalis and \\
\hline & & & & & & kidney complaints (C) & (Z) \\
\hline & & & & & & fever (C) & \\
\hline
\end{tabular}


Table 4 List of medicinal plant species reported in Zetaquira and Campo Hermoso including ethnobotanical characteristics (Continued)

\section{ALCG 374}

Llantén

Common plantain

Cymbopogon citratus (DC.) Stapf.*

herb cultivated in solar

introduced India ${ }^{2}$

$\begin{array}{ll}0.56 / & 0.44 / \\ 0.60 & 0.50\end{array}$

leaves $(\mathrm{B})^{*} 2$

Poaceae

\section{ALCG 77}

Limonaria or limoncillo

West Indian,Lemon grass,

oil grass

Rumex crispus L.

herb wild in open pastures

introduced Europe ${ }^{7}, \quad 0.08 / \quad 0.19 /$

leaves (B)

West Asia ${ }^{20}$

Polygonaceae

ALCG 148

Romaza, Lengua de vaca

Curled dock

Rubus glaucus Benth.

sub-shrub
cultivated in

homegarden

$\begin{array}{lll}\text { native from Mexico } & 0.28 / & 0.50 /\end{array}$

to Ecuador and from

center to south of

Colombian Andeans ${ }^{7}$

shoots and fruits (B)

shoots (Z)

Moras

Andean blackberry

Citrus aurantium var. Amara L.

small tree
cultivated in homegarden introduced Southern $\quad 0.20 / \quad 0.69 /$ Vietnam $^{13}$, Southeast $\quad 0.20 \quad 0.25$ Asia $^{5}$ rheumatism (B)

against skin infections and inflamations (C)

against rashes $(C)$

against spots (C)

caughs ( $Z$ )

healing wounds (Z)

intestine complaints (Z)

gripe (Z)

fevers (C)

fevers (B)

kidney complaints (C)

liver cleanser (C)

intestinal inflamations (Z)

infusion or decoction/ oral (B)

infusion/ oral (Z)

decoction/ oral (Z)

decoction/ oral (C)

fresh extract by maceration/ oral (C)

fresh extract by maceration/ topical-baths (Z) fresh extract by maceration/ oral (C)

heated leaves/topical directly on the lower belly (Z)

decoction/ oral and decoction/ topical-baths (B)

decoction/topical-baths and infusion/ oral (C)

decoction/topical-baths (C)

decoction/ oral (Z)

relaxant (B)

steam baths/ topical (B)

infusion/ oral (C)

decoction/ topical-for rinsing mouth (B)

steam baths/ topical (C) 
Table 4 List of medicinal plant species reported in Zetaquira and Campo Hermoso including ethnobotanical characteristics (Continued)

\begin{tabular}{|c|c|c|c|c|c|c|c|}
\hline & & & & & & & decoction/ topical-baths (Z) \\
\hline & & & & & & mouth infections (B) & decoction/ oral (Z) \\
\hline & & & & & & rheumatism (B) & \\
\hline Rutaceae & & & & & leaves $(Z)$ & body dryness (Z) & \\
\hline \multicolumn{8}{|l|}{ Naranjo agrio } \\
\hline \multicolumn{8}{|l|}{$\begin{array}{l}\text { Bitter orange,Seville } \\
\text { orange }\end{array}$} \\
\hline \multirow[t]{9}{*}{$\begin{array}{l}\text { Citrus maxima (Burm. ex } \\
\text { Rumph.) Merr. }\end{array}$} & \multirow{9}{*}{$\begin{array}{l}\text { small tree } \\
\text { cultivated in } \\
\text { homegarden }\end{array}$} & \multirow[t]{9}{*}{$\begin{array}{l}\text { introduced South } \\
\text { East Asia } 5,13\end{array}$} & \multirow[t]{9}{*}{$\begin{array}{l}0.36 / \\
0.24\end{array}$} & \multirow[t]{9}{*}{$\begin{array}{l}0.25 / \\
0.19\end{array}$} & \multirow[t]{5}{*}{ fruits (B) } & gripe (B) & \multirow{3}{*}{$\begin{array}{l}\text { juice,alone or in combination with } \\
\text { aguadepanela-U3-/ oral (B) } \\
\text { decoction of shoots/ oral (C) } \\
\text { juice in combination with water / oral (B) }\end{array}$} \\
\hline & & & & & & \multirow[t]{2}{*}{ stomach ache (B) } & \\
\hline & & & & & & & \\
\hline & & & & & & \multirow[t]{2}{*}{ inflamations because infections (C) } & \multirow{6}{*}{$\begin{array}{l}\text { decoction / topical-baths (C) } \\
\text { juice in combination with water/ oral (Z) } \\
\text { decoction/ topical-baths (Z) }\end{array}$} \\
\hline & & & & & & & \\
\hline & & & & & shoots (C) & headache (Z) & \\
\hline & & & & & & fevers (Z) & \\
\hline & & & & & fruits (B) & \multirow[t]{2}{*}{ rheumatism (Z) } & \\
\hline & & & & & fruits and branches $(\mathrm{C})$ & & \\
\hline Rutaceae & & & & & fruits (Z) & & \\
\hline ALCG 98 & & & & & fruits and branches (Z) & & \\
\hline \multicolumn{8}{|l|}{ Limón mandarin } \\
\hline \multicolumn{8}{|l|}{ Pommelo, Pumelo } \\
\hline \multirow[t]{4}{*}{ Ruta graveolens L.* } & \multirow[t]{8}{*}{$\begin{array}{l}\text { herb cultivated } \\
\text { in small garden }\end{array}$} & \multirow{4}{*}{$\begin{array}{l}\text { introduced Canarian } \\
\text { Islands Europe } \text { meridional }^{5}\end{array}$} & \multirow[t]{4}{*}{$\begin{array}{l}1.24 / \\
1.08\end{array}$} & \multirow[t]{4}{*}{$\begin{array}{l}0.75 / \\
0.50\end{array}$} & \multirow[t]{4}{*}{ leaves (B) } & \multirow[t]{4}{*}{ to strengthen uterus (B) } & $\begin{array}{l}\text { fresh choped leaves combinedwith boiled } \\
\text { egg/ oral (B) }\end{array}$ \\
\hline & & & & & & & decoction/ oral (B) \\
\hline & & & & & & & decoction/ topical-bath (Z) \\
\hline & & & & & & & fresh extract by maceration in water/ oral (C) \\
\hline \multirow[t]{4}{*}{ Rutaceae } & & & & & \multirow[t]{3}{*}{$\begin{array}{l}\text { leaves, flowers and } \\
\text { stems }(B)^{*} 2\end{array}$} & $\begin{array}{l}\text { against cramps in uterus and } \\
\text { menstural pains (B) }\end{array}$ & \multirow[t]{4}{*}{ infusion/ oral (Z) } \\
\hline & & & & & & rheumatism (Z) & \\
\hline & & & & & & intestinal parasits $(\mathrm{C})$ & \\
\hline & & & & & leaves and stems (C) & cardioregulator (Z) & \\
\hline ALCG 86 & & & & & leaves (Z) & & \\
\hline \multicolumn{8}{|l|}{ Ruda } \\
\hline $\begin{array}{l}\text { Citrus limon (L.) Burm. F. } \\
(\mathrm{e}-\mathrm{Z})\end{array}$ & $\begin{array}{l}\text { small tree } \\
\text { cultivated in } \\
\text { homegarden }\end{array}$ & $\begin{array}{l}\text { introduced Himalaya } \\
\text { and Indochina }\end{array}$ & $\begin{array}{l}0.52 / \\
0.20\end{array}$ & $\begin{array}{l}1.00 / \\
0.63\end{array}$ & fruits (B) & gripe (B) & $\begin{array}{l}\text { juice alone or in combination with water or } \\
\text { aguadepanela/ oral (B) }\end{array}$ \\
\hline
\end{tabular}


Table 4 List of medicinal plant species reported in Zetaquira and Campo Hermoso including ethnobotanical characteristics (Continued)

\begin{tabular}{|c|c|c|c|c|c|c|c|}
\hline & & & & & & fevers (B) & juice in combination with water/ oral (B) \\
\hline & & & & & & against infections in throat (B) & juice/ to gargle-topical (B) \\
\hline & & & & & & & juice in combination with water/ oral (B) \\
\hline \multirow[t]{2}{*}{ Rutaceae } & & & & & & stomach complaints (B) & $\begin{array}{l}\text { a fresh hitted-hot fruit/ to massage-topical } \\
\text { (C) }\end{array}$ \\
\hline & & & & & & & $\begin{array}{l}\text { fruit pieces mixed with salt in water/ to do } \\
\text { baths-topical (C) }\end{array}$ \\
\hline ALCG 93 & & & & & & intestine complaints (B) & fresh fruit pulp/ to rub the head-topical (C) \\
\hline \multirow[t]{3}{*}{ Limón ácido } & & & & & & rheumatism (C) & juice in combination with water/ oral (Z) \\
\hline & & & & & & againts excema $(C)$ & \\
\hline & & & & & & headache (C) & \\
\hline Lemon, Citrus & & & & & & to prevent high blood presure (Z) & \\
\hline Physalis peruviana $\mathrm{L}$. & $\begin{array}{l}\text { shrub cultivated } \\
\text { in homegarden }\end{array}$ & $\begin{array}{l}\text { introduced Northern } \\
\text { South America-Peru }\end{array}$ & $\begin{array}{l}0.16 / \\
0.04\end{array}$ & $\begin{array}{l}0.19 / \\
0.00\end{array}$ & fruits (B) & eye dust and infections (B) & $\begin{array}{l}\text { fresh extract from maceration/ topical-eye } \\
\text { drops (B) }\end{array}$ \\
\hline Solanaceae & & & & & & & a variation is a concoction with honey $(\mathrm{C})$ \\
\hline \multicolumn{8}{|l|}{ ALCG 163} \\
\hline \multicolumn{8}{|l|}{ Uchuba,Guchuba } \\
\hline \multicolumn{8}{|l|}{ Cape gooseberry } \\
\hline \multirow[t]{3}{*}{ Solanum nigrum L. * } & \multirow{3}{*}{$\begin{array}{l}\text { sub shrub } \\
\text { spontaneous in } \\
\text { solar }\end{array}$} & \multirow{3}{*}{$\underset{13,23}{\text { introduced Eurasia }}$} & \multirow{3}{*}{$\begin{array}{l}0.20 / \\
0.04\end{array}$} & \multirow{3}{*}{$\begin{array}{l}0.19 / \\
0.00\end{array}$} & \multirow[t]{3}{*}{ fruits and leaves $(B)^{*} 2$} & diabetes $(C)$ & infusion/ oral (C) \\
\hline & & & & & & & decoction/ topical-baths (Z) \\
\hline & & & & & & inflamations and skin infections (Z) & concentrated extract by decoction/ oral (Z) \\
\hline Solanaceae & & & & & & respiratory complaints (Z) & \\
\hline \multicolumn{8}{|l|}{ ALCG 157} \\
\hline \multicolumn{8}{|l|}{ Yerbamora(Zetaquira) } \\
\hline \multicolumn{8}{|l|}{$\begin{array}{l}\text { Almoraduz(Campo } \\
\text { Hermoso) }\end{array}$} \\
\hline \multicolumn{8}{|l|}{ black night shade } \\
\hline \multirow[t]{3}{*}{$\begin{array}{l}\text { Cestrum mariquitense } \\
\text { Kunth }\end{array}$} & \multirow[t]{3}{*}{$\begin{array}{l}\text { shrub cultivated } \\
\text { in solar }\end{array}$} & \multirow{3}{*}{$\begin{array}{l}\text { native Tropical } \\
\text { America }{ }^{13} \text { Boyacá } \\
\text { and Cundinamarca- } \\
\text { Colombia }^{3}\end{array}$} & \multirow[t]{3}{*}{$\begin{array}{l}0.08 / \\
0.08\end{array}$} & \multirow[t]{3}{*}{$\begin{array}{l}0.50 / \\
0.25\end{array}$} & \multirow[t]{3}{*}{ leaves (B) } & sinucitis (B) & $\begin{array}{l}\text { fresh extract by maceration/ topical-noise } \\
\text { drops (B) }\end{array}$ \\
\hline & & & & & & fevers (B) & fresh extract by maceration/ oral (B) \\
\hline & & & & & & headache (C) & $\begin{array}{l}\text { concoction with Hibiscus rosa sinenesis in } \\
\text { decoction/topical-baths (C) }\end{array}$ \\
\hline Solanaceae & & & & & & & fresh extract by maceration/ oral (C \\
\hline
\end{tabular}


Table 4 List of medicinal plant species reported in Zetaquira and Campo Hermoso including ethnobotanical characteristics (Continued)

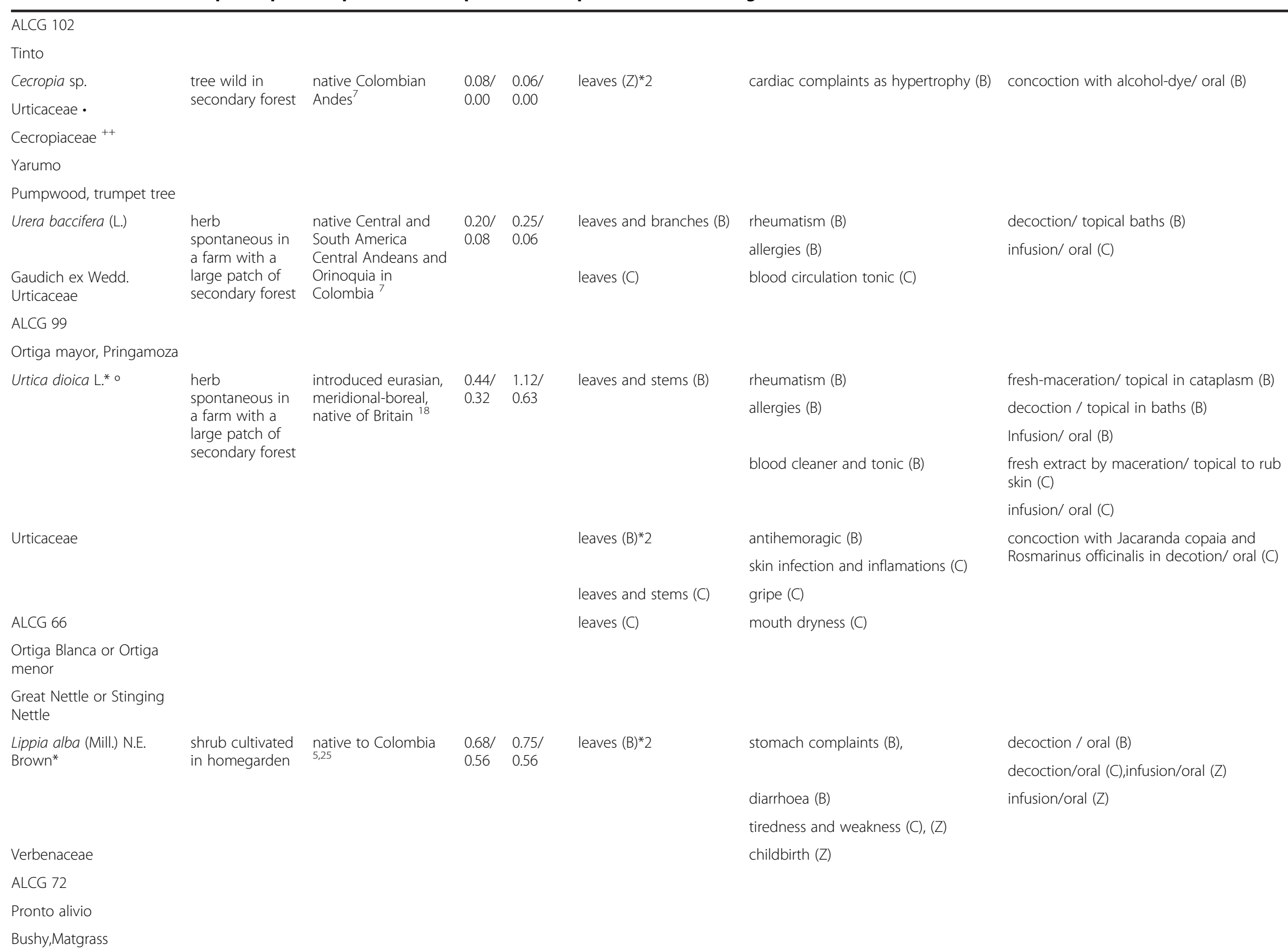


Table 4 List of medicinal plant species reported in Zetaquira and Campo Hermoso including ethnobotanical characteristics (Continued)

Lippia citriodora (Lam.)

Kunth*

native Colombian

0.25

$0.36-0.13$

leaves $(B)^{*} 2$

Verbenaceae

ALCG 70

Cidrón

Lemon Scented Verbena

Lantana camara L.

$$
\begin{array}{ll}
\text { herb wild in } & \text { native to Caribean } \\
\text { open pastures } & \text { territories including } \\
& \text { Colombia, } \\
& \text { Continental US and } \\
& \text { Hawai }{ }^{20}
\end{array}
$$

\section{$0.20 / \quad 0.25 /$ \\ leaves and stems $(B)$}

$0.08 \quad 0.25$

hepatitis (C)

yellow fever (C)

flowers (C)

flowers, stems and

leaves (C)

leaves and stems ( $Z$ )

Florota

Cambara de Espinto or

Pricky Lantana

Verbena littoralis Kunth *

herb cultivated

in solar

native western and

ranges ${ }^{5}$

$\begin{array}{ll}0.96 / & 0.88 / \\ 0.40 & 0.63\end{array}$

leaves and stems (B)

evers (B)

gripe (B)

rheumatism (Z)

rheumatism (C)

Verbenaceae

ALCG 79

Verbena blanca

Vervain, owi

Viola odorata L.*

Violaceae
Acaule herb

Cultivated in small garden introduced Europe,

Boreal Africa $^{5}$
Austral Asia and
$0.04 / \quad 0.00 /$ $0.00 \quad 0.00$ leaves*2 and stems (Z)

cough (Z)

asthma (Z) decoction/ topical-baths (B)

infusion/ oral (C)

decoction in milk/ oral and decoction/ topical-baths (C)

decoction/topical-baths (Z)

decoction/ topical baths (B)

fresh extract by maceration in water/ oral (Z)

concoction with Calendula officinalis and Piper hispidum in decoction/ topical baths (C)

concoction with Piper hispidum in decoction/ topical in baths (C)

infusion/ oral (Z)

infusion/oral (Z) 
Sweet violet/common

violet

\section{B: Both municipalities; C: Campo Hermoso; Z: Zetaquira.}

A glass bottle is filled with plant(s) parts, red grape wine usually and, it is buried under the ground during a month.

${ }^{\mathrm{U} 2} \mathrm{~A}$ similar process as it is described in U1, with the variation of the use of aguardiente, which is the introduced alcoholic drink similar to spirit (snaps).

U3 Aguadepanela is a traditional colombian homemade drink.The ingredients are water and panela, that is an unrefined food product of which the main component is sugarcane juice.

* Reported in Colombian Vademecum, 2008;* ${ }^{2}$ Plant material of interest reported in Colombian Vademecum, 2008.

Reported in WHO,2009; ${ }^{2}$ Plant material of interest reported in WHO, 2009

- www.tropicos.org.

++ www.ipni.org/ipni/plantnamesearchpage.do.

(e-CH/Z) Endangered plant species according to the perceptions of the locals in Campo Hermoso (CH) and/or Zetaquira (Z).

1 Rothmaler Werner, 1994.

Antolinez González J. C. et.al ,2008.

${ }^{3}$ Garcia Barriga Tomo III1992.

${ }^{4}$ De Fraume Melida, 1988.

${ }^{5}$ Garcia Barriga Tomo II, 1992

${ }^{6}$ Fonnegra,2007.

7 Garcia Barriga Tomo I 1992

${ }^{8}$ Perez-Arbelaez 1978.

${ }^{9}$ Centro de Educacion No Formal FUNIBA.

${ }^{10}$ Carlos Paez Perez, 1964.

${ }_{11}^{1}$ www.Botanical.com.

${ }^{12}$ www.zipcodezoo.com/plants/.

${ }_{13}$ www.en.wikipedia.org.

${ }_{14}$ www.mansfeld.ipk-gatersleben.de.

15 www.mansfeld.ipk-gaters
wwwersofindia.net.

${ }_{16}^{15}$ wwww.mobot.org.

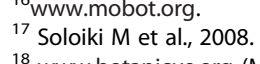

${ }_{18}^{18}$ www.botanicus.org (Missouri Botanical Garden).

${ }^{19}$ www.issg.org (global invasive species database)

${ }^{20}$ www.cbif.gc.ca/itis (Integr. Taxonomi Inf. Sys.).

${ }_{21}^{21}$ Missouri Bot. Garden 2007 in Colomb. Vademecum.

22 a.o. (tropicalforages.info).

$23 \mathrm{http}: / /$ plants.usda.gov/java/profile?symbol=SEOB4\#

${ }^{24} \mathrm{http}: / /$ www.conifers.org/cu/cup/lusitanica.htm.

${ }^{25}$ Vera et. al. in Revista Cubana de plantas medicinales 2010.

${ }^{26} \mathrm{http}: / /$ zipcodezoo.com/Key/Plantae/Cecropia_Genus.asp.

$27 \mathrm{http} / / /$ plants.jstor.org/flora/flos 003208. 
Table 5 Plant species reported as disappeared or endangered within the municipalities of Campo Hermoso and Zetaquira

\begin{tabular}{|c|c|c|}
\hline & Campo Hermoso & Zetaquira \\
\hline \multirow{8}{*}{$\begin{array}{l}\text { Disappeared } \\
\text { species }\end{array}$} & Brownea ariza & Fraxinus udhei \\
\hline & Ananas comosus & Schizolobium parahybum \\
\hline & Parietaria officinalis & $\begin{array}{l}\text { Chrysophyllum } \\
\text { colombianum }\end{array}$ \\
\hline & Furcraea sp. & 'Alma negra' \\
\hline & Malva sp. & \\
\hline & 'Gualola' & \\
\hline & 'Mano de León' & \\
\hline & 'Bejuco de roca' & \\
\hline \multirow{6}{*}{$\begin{array}{l}\text { Endangered } \\
\text { Species }\end{array}$} & Furcraeae macrophylla & Citrus limon \\
\hline & Artemisia absinthium & Saccharum officinarum \\
\hline & $\begin{array}{l}\text { Ocimum } \\
\text { campechianum }\end{array}$ & Juglans neotropica \\
\hline & Juglans neotropica & Rosmarinus officinalis \\
\hline & Ceroxylum quindiuense & \\
\hline & Cedrela spp. & \\
\hline
\end{tabular}

Wilcoxon-Mann-Whitney tests $[16,17]$ were used to test for differences between introduced and native medicinal plant species in their estimated and actual UV within the two municipalities.

Finally, similarities of use of introduced and native medicinal plant species between the municipalities were calculated using the Jaccard Index [21,22].

\section{Results}

\section{Medicinal plant species and plant characteristics}

Interviews and questionnaires supplied a list of a total of 80 medicinal plant species used in the treatments of ailments within the municipalities of Campo Hermoso and Zetaquira. Of these, 78 species were taxonomically identified. Results are based on the 78 identified species of which 35 were native species and 43 introduced (Table 4), belonging to 74 genera and 41 floristic families. The families with the highest numbers of species reported as medicinal were: Asteraceae (10 species), Lamiaceae (7), Apiaceae, Rutaceae and Verbenaceae (each 4), and Malvaceae, Solanaceae and Urticaceae (each 3).

Twenty-nine of the identified species and the genus Cecropia Loefl. were included in the list of medicinal plants evaluated and accepted in the Colombian pharmacopeia [23]. Thirteen species were reported in the World Health Organization's (WHO) monographs on selected medicinal plants [24] (Table 4).

During interviews and meetings interviewees were able to reflect about the local resources of medicinal plant species, their importance and, additionally, to discuss

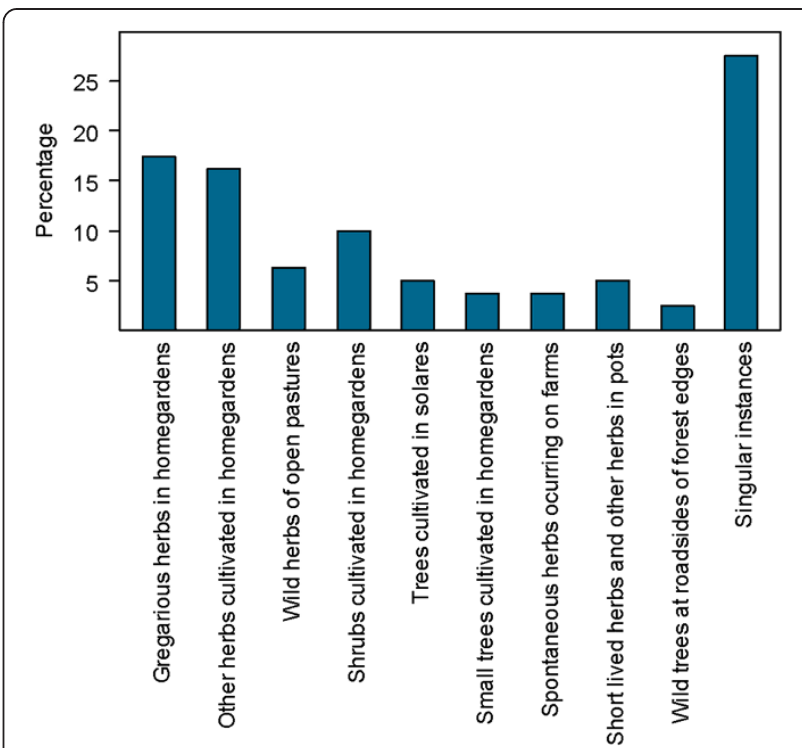

Figure 2 Life form and habitats of medicinal plant species reported in Campo Hermoso and Zetaquira. Gregarious herbs in home gardens refers to cultivated plants; Home gardens include also solares; Short-lived herbs and other herbs in pots are also cultivated; singular instances refers to: a wild twining plant along riverbanks, a succulent tall herb on farmland, a wild vine at shady and moist edges of secondary forests, a succulent plant in pots, a tree cultivated in garden.

factors that may have increased or reduced plant diversity, such as logging, over- exploitation and difficulties of cultivation. Some interviewees reported that they found it difficult to treat certain diseases because they failed to find the plant species needed. An example is Brownea ariza Benth., a native species, which locals consider

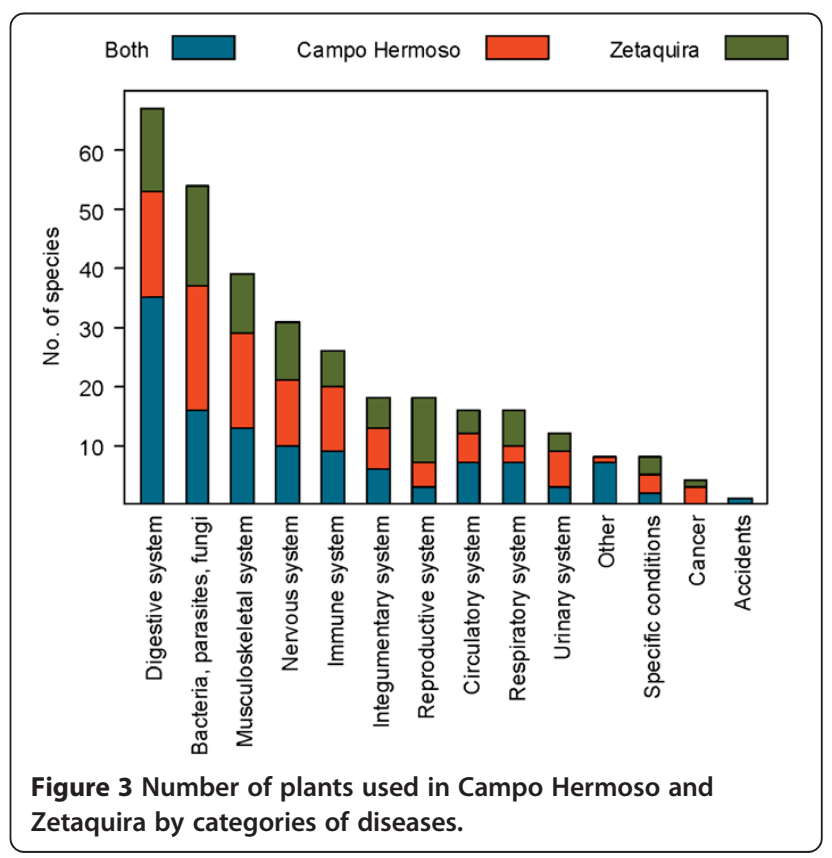




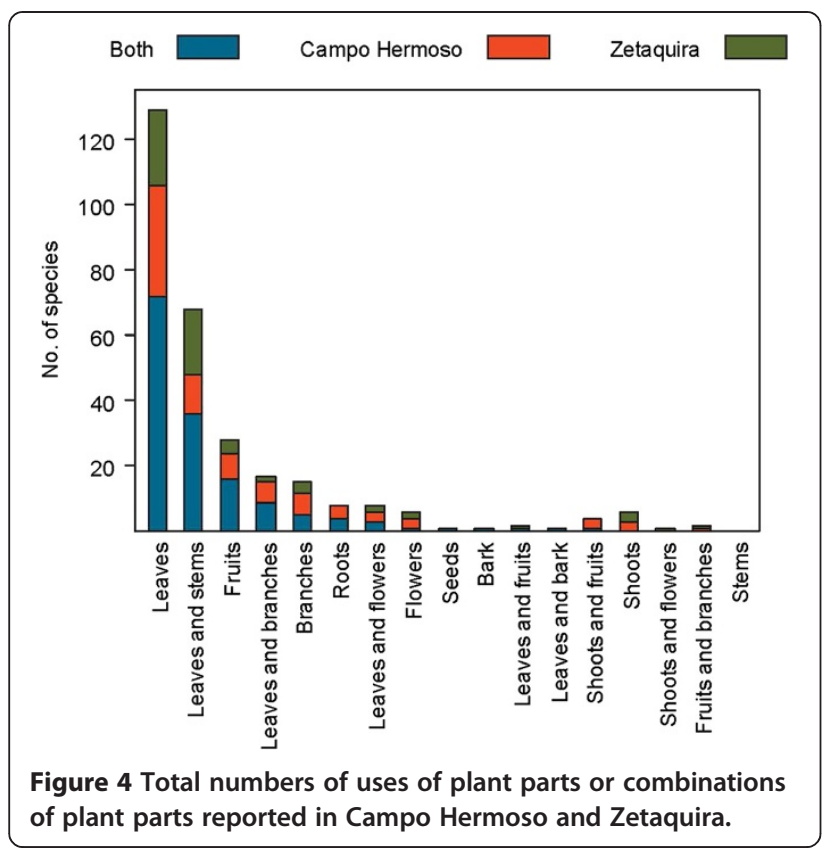

extinct in the Campo Hermoso area. This species was used as a haemostat, i.e. to stop bleeding, and as laxative. Another example is Juglans neotropica Diels., a native species, used as fungicide and bactericide, which was reported to be endangered in both municipalities (Table 4 and 5). Due to the lack of samples it was not possible to scientifically identify all the species reported as threatened and disappearing by the interviewees.

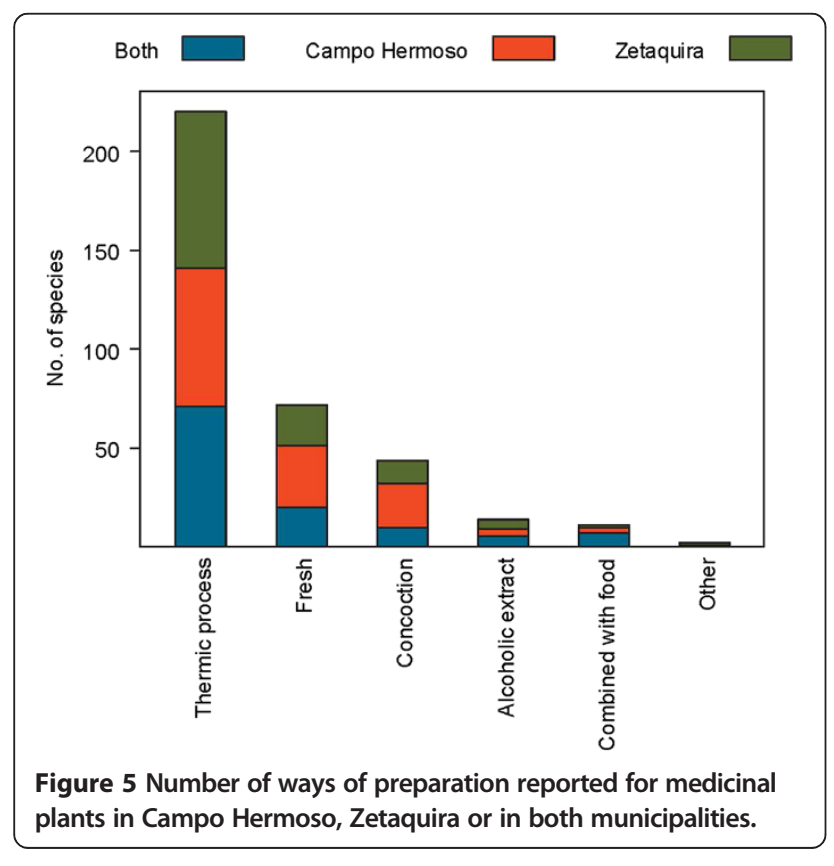

According to informants' reports and the subsequent calculations of average UVs the most popular medicinal species, in the municipality of Campo Hermoso were Urtica dioica (0.88), Jaccaranda copaia (0.81) and Citrus limon (0.81) while in Zetaquira the most popular species were Ruta graveolens (average UV=1.16), Melissa officinalis (0.8), and Aloe vera (0.6). Across both municipalities, the most common and popular medicinal plant species were Mentha suaveolens, Ambrosia cumanensis Kunth, and Verbena littoralis (Table 4).

In relation to life form and habitat, the collected plant species could be assigned to 10 categories shown in the Figure 2 (Table 4). Of the native species $40 \%$ were found in natural habitats whereas $27 \%$ of the introduced species were found as naturalised in the wild.

The naturally occurring and naturalised species were distributed widely within families. For example, Asteraceae ( 2 herbs), Acanthaceae (1 tree). Aristolochiaceae (1 twiner) and Commelinaceae (2 vines) (Table 4).

\section{Traditional plant use in Campo Hermoso and Zetaquira}

Traditional plant uses in the municipalities of Campo Hermoso and Zetaquira are represented in Figures 3, 4, 5 , 6. The total number of medicinal plants uses described by informants was higher in more remote Campo Hermoso than in Zetaquira.

Common diseases, such as colds, but also a small number of difficult or complicated diseases, such as cancer, were reported to be treated. The majority of plants mentioned by the informants were reported to be effective in curing the diseases they were applied to. Additionally, most of the informants mentioned the importance

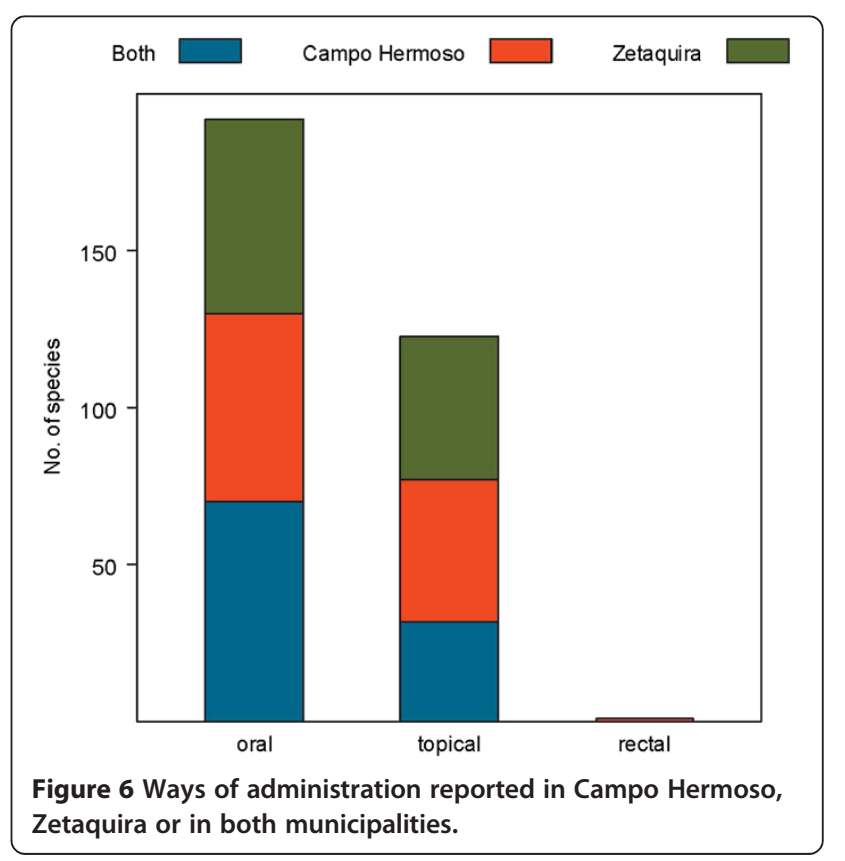


of using folk traditions, e.g. prayers, during treatments in order to ensure the effect of the medicinal plant.

The diseases described by interviewees were grouped in 14 categories (Table 2). The five categories of ailments/diseases with the highest numbers of plants reported in both municipalities were associated with: the digestive system, infections, musculoskeletal, the nervous system, and the immune system. The corresponding most popular medicinal plant species are shown in Table 6. Significant dissimilarities between municipalities occurred in treatments of musculoskeletal, immune and urinary systems, and cancer where the total numbers of plants mentioned in more remote Campo Hermoso was almost double the number of Zetaquira. The category with the highest number of medicinal plant species reported from Zetaquira was 'reproductive system' with 11 plants. There were no plant species specifically reported for the treatment of the two parasitic, epidemic, local diseases of Chagas (caused by Trypanosoma cruzi) and Malaria (Paludism). Only two species, Cattleya schroederae Rchb.f. and Jaccaranda cf. copaia, were reported for the treatment of Dengue, a viral, epidemic mosquito transmitted disease also affecting these populations (Table 4).

Regarding the plant parts used, leaves were the plant part most frequently reported for remedy preparations, in both municipalities. Other commonly used plant parts were, in descending order of total counts in both municipalities: combination of leaves with stems (36), fruits (16), combination of leaves with branches (9), branches (5), roots (4),

\begin{tabular}{|c|c|c|}
\hline Disease category & $\begin{array}{l}\text { No. of plants reported } \\
\text { in both municipalities }\end{array}$ & $\begin{array}{l}\text { Popular plant } \\
\text { species used in } \\
\text { treatments }\end{array}$ \\
\hline \multirow[t]{3}{*}{ Digestive system } & 35 & Melissa officinalis \\
\hline & & Cymbopogon citratus \\
\hline & & Apium graveolens \\
\hline \multirow{3}{*}{$\begin{array}{l}\text { Infections from } \\
\text { bacteria, parasites or } \\
\text { fungi }\end{array}$} & 16 & Mentha suaveolens \\
\hline & & Citrus limon \\
\hline & & Jaccaranda copaia \\
\hline \multirow{3}{*}{$\begin{array}{l}\text { Muskuloskeletal } \\
\text { system }\end{array}$} & 13 & Verbena littoralis \\
\hline & & Urtica dioica \\
\hline & & Aristolochia ringens \\
\hline \multirow[t]{3}{*}{ Nervous system } & 10 & Melissa officinalis \\
\hline & & Matricaria chamomilla \\
\hline & & Lippia citriodora \\
\hline \multirow[t]{3}{*}{ Immune system } & 9 & Aloe vera \\
\hline & & Melissa officinalis \\
\hline & & Verbena littoralis \\
\hline
\end{tabular}

and combination of leaves with flowers (3). Other combinations of different plant parts were mentioned only rarely. The use of seeds was not popular in either of the municipalities (Figure 4).

In both municipalities, similar numbers of applications were reported for Cymbopogon citratus, Plantago major, Petroselinum crispum and Lippia alba (Mill.) N.E.Br. These species received the highest scores for application of leaves. Most popular species with the highest applications of leaves were: Cattleya schroederae (4) and Piper cf. bogotense C.DC. (3), in Campo Hermoso, and Origanum majorana L. (3) in Zetaquira (Table 4).

Eighty different ways of preparation of remedies with medicinal plant species were described. They were grouped according to the type of processing: thermic methods, alcoholic extracts or fresh use. Other ways of preparation of the remedies, such as the combination with food and with other plants (concoction) were also mentioned and thus counted. Practices according to beliefs included the effect of moon or sunlight, esoteric ways and specific ways to treat pets. These additional ways of preparing traditional remedies were included in one group named 'other' (Figure 5).

The most popular way of administration remedies was oral followed by the topical in both municipalities (Figure 6). Among the most popular topical ways of administration, 'baths' yielded the highest scores (13 counts) in both municipalities, 26 in Campo Hermoso and 25 in Zetaquira. Massage was the second most popular in Campo Hermoso, while 'emplast' was the second most popular in Zetaquira.

Healers and amateur healers unanimously reported that bitter plants were denominated 'hot plants' ('plantas calientes', in Spanish), while plants with sweet flavour were denominated 'cold plants' ('plantas frias'). These terms suggested the level of caution with which the medicinal plants should be used. Bitter plants should be used in small doses at low frequency because most of them can produce eye or heart problems. Bitter plants should be used orally only in situations caused by musculoskeletal complaints. Further, they recommended to initially use topical treatments and, in the most general sense, to follow a diet when using medicinal plants. Popular plants used for the purposes outlined above in the localities are described in Table 4.

Table 7 shows the plant species with the highest values obtained in aUV according to the sum of the values from both municipalities. In addition, eUV are also included.

\section{Differences and similarities among plant groups and families}

UVs of introduced plant species were significantly higher than native species in the more accessible municipality of Zetaquira (Table 8), while there were no significant 
Table 7 Plant species according to the highest actual index use values obtained in both municipalities

\begin{tabular}{|c|c|c|c|c|c|c|c|}
\hline Scientific name (Voucher number) & $\begin{array}{l}\text { alUV } \\
\text { all }\end{array}$ & alUV & alUV & $\begin{array}{l}\text { elUV } \\
\text { all }\end{array}$ & elUV & elUV & Medicinal uses \\
\hline Mentha suaveolens Ehrh. * (ALCG 127) & 1 & 1.04 & 0.94 & 1.10 & 1.20 & 0.94 & $\begin{array}{l}\text { stomach ache(B), headaches(B), intestinal inflammations(B), } \\
\text { intestinal parasites(Z), fevers(Z), menstrual cramps(Z), diaper } \\
\text { rash(Z) }\end{array}$ \\
\hline Ruta graveolens L. *(ALCG 86) & 0.85 & 1.08 & 0.50 & 1.05 & 1.24 & 0.75 & $\begin{array}{l}\text { against cramps in uterus and menstrual pains(B), } \\
\text { rheumatism(Z), intestinal parasites }(C) \text {, cardio-regulator(Z) }\end{array}$ \\
\hline Melissa officinalis L. * (ALCG 92) & 0.68 & 0.72 & 0.63 & 0.83 & 0.88 & 0.75 & $\begin{array}{l}\text { gripe(B),fevers(B),relaxant(B),stomach ache(B), intestine } \\
\text { complaints(B) }\end{array}$ \\
\hline Aloe vera (L.) Burman $\mathrm{f} .{ }^{*}{ }^{\circ}$ & 0.6 & 0.63 & 0.63 & 0.64 & 0.64 & 0.63 & $\begin{array}{l}\text { asthma (B), cough(B), fever(B), headache }(B) \text {, healing wounds } \\
\text { and skin spots(B), external inflammations(B), hair treatment } \\
\text { (B), stomach cancer(Z) }\end{array}$ \\
\hline Ambrosia cumanensis Kunth (ALCG 63) & 0.4 & 0.81 & 0.98 & 0.88 & 0.88 & 1.13 & $\begin{array}{l}\text { gripe(B), rheumatism(B), children bladder complaints(B), } \\
\text { menstrual cramps(Z), colds and spams in muscles(C), } \\
\text { stomach ache(C), intestinal complaints(C), against flies(Z) }\end{array}$ \\
\hline Lippia alba (Mill.) N.E.Br. (ALCG 72) & 0.56 & 0.56 & 0.71 & 0.68 & 0.68 & 0.75 & $\begin{array}{l}\text { stomach complaints(B), diarrhoea(B), tiredness and } \\
\text { weakness }(C) /(Z) \text {, childbirth(Z) }\end{array}$ \\
\hline $\begin{array}{l}\text { Cymbopogon citratus (DC.) Stapf. * } \\
\text { (ALCG 77) }\end{array}$ & 0.6 & 0.50 & 0.51 & 0.56 & 0.56 & 0.44 & $\begin{array}{l}\text { stomach aches(B), intestine complaints }(Z) \text {, gripe }(Z) \text {, prostate } \\
\text { complaints }(C) \text {, fevers }(C)\end{array}$ \\
\hline Verbena littoralis Kunth (ALCG 79) & 0.4 & 0.63 & 0.93 & 0.96 & 0.96 & 0.88 & $\begin{array}{l}\text { fevers }(B) \text {, gripe }(B) \text {, rheumatism }(Z) /(C) \text {, tiredness and } \\
\text { weakness }(C)\end{array}$ \\
\hline Urtica dioica L. * (ALCG 66) & 0.32 & 0.63 & 0.71 & 0.44 & 0.44 & 1.13 & $\begin{array}{l}\text { rheumatism }(B) \text {, allergies }(B) \text {, blood cleaner and tonic }(B) \text {, } \\
\text { anti-haemorrhagic }(B) \text {, skin infection and inflammations }(C) \text {, } \\
\text { gripe }(C) \text {, mouth dryness }(C)\end{array}$ \\
\hline Citrus limon (L.) Burm. F. * (ALCG 93) & 0.2 & 0.63 & 0.71 & 0.52 & 0.52 & 1.00 & $\begin{array}{l}\text { gripe }(B) \text {, fevers }(B) \text {, infections in throat }(B) \text {, stomach and } \\
\text { intestine complaints(B), rheumatism }(C) \text {, eczema }(C) \text {, } \\
\text { headache }(C) \text {, to prevent high blood pressure }(\mathrm{Z})\end{array}$ \\
\hline $\begin{array}{l}\text { Jacaranda cf. copaia (Aubl.) D.Don } \\
\text { (ALCG 69) }\end{array}$ & 0.12 & 0.69 & 0.49 & 0.2 & 0.2 & 0.94 & $\begin{array}{l}\text { Intestinal complaints(B), Kidney complaints(Z), rheumatism } \\
(C) \text {, gripe }(C) \text {, fevers }(C) \text {, dengue }(C) \text {, skin infections(C), } \\
\text { circulatory complaints(C), mouth dryness }(C)\end{array}$ \\
\hline Matricaria chamomilla L. *(ALCG 96) & 0.32 & 0.38 & 0.34 & 0.32 & 0.32 & 0.38 & $\begin{array}{l}\text { stomach-complaints(B),relaxant(B), rheumatism(Z), colds, } \\
\text { spams and cramps in muscles (C) }\end{array}$ \\
\hline Rosmarinus officinalis L. ${ }^{*}$ (ALCG 149) & 0.44 & 0.19 & 0.39 & 0.44 & 0.44 & 0.31 & $\begin{array}{l}\text { lungs complaints(B), tooth ache(B), hypertension } \\
\text { complaints(B), against hair loss(B), muscles pain (C) } \\
\text { rheumatism(C), stomach ache(Z), tiredness(Z) }\end{array}$ \\
\hline $\begin{array}{l}\text { Petroselinum crispum (Mill. Nyman ex A. } \\
\text { W. Hill*(ALCG 87) }\end{array}$ & 0.36 & 0.25 & 0.46 & 0.44 & 0.44 & 0.50 & $\begin{array}{l}\text { cardiovascular complaints as high blood pressure(B), } \\
\text { enhance stomach function(B), halitosis(B), condiment herb } \\
\text { (B), kidney complaints(C), intestinal pain(C), against } \\
\text { menstrual pain(Z) }\end{array}$ \\
\hline Lippia citriodora (Lam.) Kunth (ALCG70) & 0.36 & 0.13 & 0.39 & 0.48 & 0.48 & 0.25 & $\begin{array}{l}\text { stomach and intestinal complaints(B), relaxant(B), general } \\
\text { indisposition(Z) }\end{array}$ \\
\hline Mentha viridis L. * (ALCG 91) & 0.2 & 0.38 & 0.41 & 0.36 & 0.36 & 0.50 & gripe(B), stomach ache(B), inflammations of intestine(B) \\
\hline Sambucus nigra L. *(ALCG 68) & 0.24 & 0.25 & 0.66 & 0.72 & 0.72 & 0.56 & $\begin{array}{l}\text { gripe(B), eye inflammations and infections(B), fevers(B), } \\
\text { tiredness and weakness(Z), respiratory, complaints(B), head } \\
\text { ache(Z), against hair lice }(C)\end{array}$ \\
\hline Cattleya schroederae Rchb.f. (ALCG 165) & 0 & 0.63 & 0.27 & 0 & 0 & 0.69 & typhoid(C), dengue(C), fever(C), intestinal complaints(C) \\
\hline Calendula officinalis L. * (ALCG 57) & 0.24 & 0.19 & 0.49 & 0.6 & 0.6 & 0.31 & $\begin{array}{l}\text { external-internal inflammations(B), gastritis(C), skin } \\
\text { infections (Z), menstrual cramps(Z) }\end{array}$ \\
\hline Ocimum campechianum Mill. (ALCG 74) & 0.16 & 0.31 & 0.41 & 0.28 & 0.28 & 0.63 & intestinal complaints(B), eye dust (B), gripe(Z) \\
\hline Chenopodium ambrosioides L. (ALCG 80) & 0.12 & 0.38 & 0.41 & 0.4 & 0.4 & 0.44 & stomach complaints(B), intestinal parasites(B) \\
\hline $\begin{array}{l}\text { Citrus maxima (Burm. ex Rumph.) Merr. } \\
\text { *(ALCG 98) }\end{array}$ & 0.24 & 0.19 & 0.32 & 0.36 & 0.36 & 0.25 & $\begin{array}{l}\text { gripe }(B) \text {, stomach ache }(B) \text {, inflammations because infections } \\
(C) \text {, headache }(Z) \text {, fevers }(Z) \text {, rheumatism }(Z)\end{array}$ \\
\hline Apium graveolens $\mathrm{L} .{ }^{*}$ 。 & 0.24 & 0.19 & 0.22 & 0.2 & 0.2 & 0.25 & $\begin{array}{l}\text { intestinal obstipation(B), stomach ache }(B) \text {, condiment herb } \\
(B) \text {, relaxant(B), to regulate menstruation(Z) }\end{array}$ \\
\hline
\end{tabular}


Table 7 Plant species according to the highest actual index use values obtained in both municipalities (Continued)

\begin{tabular}{|c|c|c|c|c|c|c|c|}
\hline Plantago major L*.(ALCG 374) & 0.16 & 0.31 & 0.51 & 0.32 & 0.32 & 0.81 & $\begin{array}{l}\text { eye dust and infections(B), gastritis(B), liver cleanser(B), } \\
\text { kidney complaints }(C) \text {, fever(C), eczema(C), healing wounds } \\
\text { (Z) }\end{array}$ \\
\hline Citrus aurantium var. amara L. ** & 0.2 & 0.25 & 0.39 & 0.2 & 0.2 & 0.69 & $\begin{array}{l}\text { relaxant(B), headache(C), mouth infections(B), rheumatism } \\
(B) \text {, body dryness(Z) }\end{array}$ \\
\hline
\end{tabular}

Includes also estimated index use values.

*Introduced plant species; ${ }^{\circ}$ No specimen collected; (C) Campo Hermoso; (Z) Zetaquira; alUV Actual Index Use Value; elUV Estimated Index Use Value; all-alUV/elUV Sum of all values corresponding to actual or estimated uses.

differences between the two groups in the more remote municipality of Campo Hermoso and nor in the two municipalities combined.

Numbers of plant species for which estimated and actual UVs could be calculated did not differ greatly between the two municipalities (Table 9). Nevertheless, totals of actual UV of introduced species were higher in Zetaquira than in Campo Hermoso. Totals of actual UV of native species were higher in Campo Hermoso than in Zetaquira. Totals of common plant species including introduced and native ones were very high in both municipalities.

There were many similarities in the use of both introduced and native plant species between the municipalities (Table 10).

Estimated Family Use Values (FUV) were higher than actual family use values in almost all cases (Table 11). Estimated and actual FUV were commonly higher for Campo Hermoso than for Zetaquira. Plant families represented by high numbers of plant species did not always have high FUVs, i.e. Asteraceae and Apiaceae. Some families, of which only one plant species was reported, surprisingly obtained high values, e.g. Aristolochiaceae which had the highest estimated use value. Another interesting case was the Asphodelaceae which yielded high actual use values. Furthermore, Asphodelaceae and Poaceae coincided in having high actual use values in both municipalities.

The list of the most popular medicinal plant species based on median of actual and estimated Use Values, among amateur healers and healers is shown in Table 12.

From the amateur healers' list, $87 \%$ species were introduced including six herbs and one shrub, while only $13 \%$, i.e. one herb, was native. In the case of healers, $57 \%$ in the list of preferred species were native, which included two trees and two shrubs, while the remaining $43 \%$ were introduced, i.e. two herbs and one vine (Table 4).

\section{Discussion}

Significance of the use of medicinal plants within the municipalities

The patterns in traditional knowledge and medicinal plant use described in this study is in accordance with other studies [25-27] in the high number of introduced species mostly herbs. Especially for the more accessible municipality of Zetaquira the use of native species was rarely reported.

Rapid adoption of introduced species in traditional medicine can be understood as a response to new opportunities arising with globalisation. Inefficacy of native species may lead to experimentation with introduced species. Introduced species enrich the arsenal of species used in treatments and are often seen as very powerful [28-30]. Adoption of introduced species may be seen as a way to reshape and re-vitalise traditional practices, which in many places provide an important alternative to the official health care services within developing countries.

The effectiveness of treatments with popular introduced plant species that are used world-wide indicates the need to promote and further the studies on the use and effectiveness of these plant species [23,24]. In the Colombian pharmacopoeia list of officially accepted plants for medicinal use $44 \%$ of the total of 149 species are introduced [23,31].

The flip-side of the coin is that with the adoption of introduced medicinal plant species peoples' interest in cultivating native species decreases. In this study most of the popular medicinal plant species (UV >0.5) were introduced with the exception of Verbena littoralis and Ambrosia cumanensis (Table 4 and 7). Furthermore, a number of native species with multiple uses, e.g. timber but also medicinally, are currently endangered or have disappeared from the regions indicating that over- exploitation has taken place [27].

Table 8 Results of Mann-Whitney-Wilcoxon tests of differences in Index Use Values (IUV) among native and introduced medicinal plants. Med. = Median; Nat. = Native; Int. = Introduced

\begin{tabular}{|c|c|c|c|c|c|c|c|c|c|}
\hline \multirow[b]{2}{*}{ Index } & \multicolumn{3}{|c|}{ Campo Hermoso } & \multicolumn{3}{|l|}{ Zetaquira } & \multicolumn{3}{|c|}{ Both Municipalities } \\
\hline & Med. Nat. & Med. Int. & $P$ & Med. Nat. & Med. Int. & $P$ & Med. Nat. & Med. Int. & $P$ \\
\hline Actual IUV & 0.13 & 0.13 & 0.677 & 0.04 & 0.14 & 0.005 & 0.09 & 0.12 & 0.095 \\
\hline Estimated IUV & 0.25 & 0.25 & 0.750 & 0.12 & 0.20 & 0.047 & 0.22 & 0.23 & 0.237 \\
\hline
\end{tabular}


Table 9 Numbers of introduced and native medicinal plant species for which estimated and actual Index Use Values could be calculated

\begin{tabular}{lllll}
\hline Origin & Index Use Value & Campo Hermoso & Zetaquira & Both \\
\hline Introduced & Actual & 4 & 10 & 25 \\
& Estimated & 2 & 3 & 38 \\
\multirow{2}{*}{ Native } & Actual & 11 & 5 & 18 \\
& Estimated & 7 & 2 & 26 \\
\hline
\end{tabular}

The high similarities between estimated and/or actual UV (> 50\%) for introduced and native plant species showed little difference in the lists of medicinal plants between the municipalities. For introduced species, similarity values were the highest (Table 10). These results are comparable to the findings of De Ameida et al. [28] who worked with rural communities in the Northeast Brazil.

Overexploitation of medicinal plant species, including a large number of native species, has been discussed extensively. Njoroge et al. [32] found that Carissa edulis (Forssk.) Vahl, a native wild species of Ethiopia [33], is a priority species used in the treatment of several ailments, especially for stomach pains, a common disorder in the Mwingi District, Kenya. Presently, this species is threatened by overuse. Similarly, other scholars have reported that plant species most popularly used by communities depend on the kind of local diseases that people face [2], which could be a parameter to identify possible species that could become endangered by overexploitation in combination with knowledge on plant parts used, harvest techniques, demand and prices.

The most frequent diseases in the study sites according to local reports from the health centre service [34] were related to the digestive system and infections caused by bacteria, parasites or fungi. The present study shows that the highest valued species were related to exactly these diseases. Of these, the top five were all introduced (Tables 4 and Table 7) confirming that communities make use of specific plant species including introduced ones.

The large number of plant species used in the treatment of complaints of the digestive system, infections and nervous system in both municipalities, is comparable to other findings from studies involving farmer communities in other localities in department of Boyacá.

\begin{tabular}{|c|c|c|}
\hline Index Use Value & Introduced & Native \\
\hline Estimated & 88 & 74 \\
\hline Actual & 64 & 53 \\
\hline
\end{tabular}

For example, Lagos [9] found that stomach-complaints occupy the category of illnesses which is treated with the highest number of medicinal plant species, followed by complaints of the nervous system. At the same time, these results are comparable to findings from other places. In the municipalities of the city of Imbituba, Santa Catarina, Brazil Zank and Hanazaki [35] found, that digestive complaints were the disease category with the highest therapeutic applications of medicinal plants. Furthermore, Neves et al. [36], who studied various groups of the community of Trás-os-Montes, Portugal, including farmers, reported that illnesses related to the digestive system were most popularly treated with medicinal plants.

\section{Life form, habitat and proximity to cities facilitate popularity of introduced species}

It has been demonstrated in several studies [28,37,38], that life form and habitat specification determine the use of medicinal plant species. The reason usually mentioned is that herbs, of which a large number are introduced, are easy to cultivate and maintain in small gardens or pots near to or in the houses. Examples of species whose popularity can be related to life form and the ease of cultivation are Ruta graveolens that has a high medicinal value in Zetaquira, and Urtica dioica that is a highly valuable herb in Campo Hermoso. Opposed to the introduction of easily cultivated herbs, a number of ecological factors diminish advantages of cultivation and maintenance of some native plant species.

Among the most popular species of amateur healers (eight species), seven species (87.5\%) were introduced, of which six were herbs and only one a shrub (Table 12). In contrast, among healers the tendency to use native species, usually trees or shrubs, is higher: $57 \%$ of their most popular species was native and included two herbs and one vine. Since healers are specialised in the use of medicinal plants, they are in most cases willing to cultivate a diversity of species, including trees and shrubs with medicinal values, in their home gardens and on other cultivated lands. Hence, cultivation could explain the popularity of native woody species within the group of healers.

The high significance of introduced plant species according to UV (Table 8) in Zetaquira closer to the capital of the department, Tunja, could be an indication of the influence of distance to modern cities and acculturation upon the adoption of new medicinal plant species. These findings are comparable to results obtained by other studies, e.g. in Brazil, Argentina and Manus Island (Papua New Guinea) $[28,39,40]$. Inhabitants of Campo Hermoso, which is more remote and follows rural traditions, have higher knowledge on medicinal plant use. Furthermore, there are more healers in Campo Hermoso. Hence, the null 
Table 11 Family Use Values (FUV) of most popular plant families calculated from estimated and actual Index Use Values of species and number of native and introduced species per family

\begin{tabular}{|c|c|c|c|c|c|c|c|c|}
\hline Family & Native & Introd. & FUV est. CH & FUV est. Zq & FUV est. Both & FUV act. CH & FUV act. Zq & FUV act. Both \\
\hline Asteraceae & 4 & 6 & 0.26 & 0.28 & 0.27 & 0.16 & 0.16 & 0.16 \\
\hline Lamiaceae & 2 & 5 & 0.48 & 0.59 & 0.55 & 0.36 & 0.43 & 0.40 \\
\hline Apiaceae & 1 & 3 & 0.22 & 0.20 & 0.21 & 0.14 & 0.19 & 0.17 \\
\hline Rutaceae & 0 & 4 & 0.67 & 0.58 & 0.62 & 0.39 & 0.43 & 0.41 \\
\hline Verbenaceae & 4 & 0 & 0.53 & 0.58 & 0.56 & 0.39 & 0.35 & 0.37 \\
\hline Malvaceae & 0 & 3 & 0.29 & 0.07 & 0.15 & 0.10 & 0.04 & 0.07 \\
\hline Solanaceae & 1 & 2 & 0.29 & 0.15 & 0.20 & 0.08 & 0.05 & 0.07 \\
\hline Urticaceae & 2 & 1 & 0.48 & 0.24 & 0.33 & 0.23 & 0.13 & 0.17 \\
\hline Acanthaceae & 2 & 0 & 0.13 & 0.40 & 0.30 & 0.00 & 0.12 & 0.08 \\
\hline Alliaceae & 0 & 2 & 0.22 & 0.22 & 0.22 & 0.13 & 0.06 & 0.09 \\
\hline Amaranthaceae & 1 & 1 & 0.44 & 0.28 & 0.34 & 0.31 & 0.14 & 0.21 \\
\hline Bignoniaceae & 2 & 0 & 0.50 & 0.10 & 0.26 & 0.38 & 0.06 & 0.18 \\
\hline Commelinaceae & 1 & 1 & 0.09 & 0.08 & 0.09 & 0.06 & 0.04 & 0.05 \\
\hline Myrtaceae & 1 & 1 & 0.44 & 0.34 & 0.38 & 0.25 & 0.04 & 0.12 \\
\hline Phytolaccaceae & 2 & 0 & 0.31 & 0.04 & 0.15 & 0.25 & 0.00 & 0.10 \\
\hline Piperaceae & 2 & 0 & 0.47 & 0.04 & 0.21 & 0.31 & 0.00 & 0.12 \\
\hline Adoxaceae & 0 & 1 & 0.56 & 0.72 & 0.66 & 0.25 & 0.24 & 0.24 \\
\hline$\underline{\text { Aristolochiaceae }}$ & 0 & 1 & 0.75 & 0.64 & 0.68 & 0.00 & 0.04 & 0.02 \\
\hline Asphodelaceae & 0 & 1 & 0.63 & 0.64 & 0.63 & 0.63 & 0.60 & 0.61 \\
\hline Plantaginaceae & 0 & 1 & 0.81 & 0.32 & 0.51 & 0.31 & 0.16 & 0.22 \\
\hline Poaceae & 0 & 1 & 0.44 & 0.56 & 0.51 & 0.50 & 0.60 & 0.56 \\
\hline
\end{tabular}

Bold $=$ plant families with higher number of species $(>2)$; italics $=$ plant families with two plant species; underlined = plant families with one species but high FUV. Est. = estimated; act. = actual; $\mathrm{CH}=$ Campo Hermoso; $\mathrm{Zq}=$ Zetaquira; both $=$ both municipalities together.

hypothesis of similarity between plant knowledge and use between municipalities is falsified.

\section{High presence of pharmacologic components in herbs and popularity of genera}

The high presence of pharmacologic components in herbs makes them attractive for treatments of different diseases [27]. Specifically the high composition of alkaloids in leaves facilitates medicinal uses as has been indicated by the popularity of these plant parts within

Table 12 Most popular species among healers and amateur healers

\begin{tabular}{ll}
\hline Amateur healers & Healers \\
\hline Calendula officinalis L. & Jacaranda copaia (Aubl.) D.Don \\
Citrus Limon (L.) Burm.F & Aristolochia ringens Vahl. \\
Petroselinum crispum (Mill.) & Ruta graveolens L. \\
Nyman ex A.W. Hill & \\
Chenopodium ambrosioides L. & Lippia alba (Mill) N.E. Brown \\
Ruta graveolens L. & Althernanthera lanceolata Benth. \\
Cymbopogon citratus (DC.) Stapf & Psidium guinense Sw. \\
Mentha suaveolens Ehrh. & Rubus glaucus Benth. \\
Allium sativum L. & \\
\hline
\end{tabular}

several communities $[9,25,27,36]$ and also in the present study. Furthermore, a number of phytochemical studies have proven remarkable alkaloid and oil content in herbal leaves [41-43] which may provide alternatives for the pharmaceutical industry. Taxa with large numbers of useful herbs contribute to the tendency of using leaves and introduced plant species. For example, the genus Mentha, one of the most popular taxa in Campo Hermoso and Zetaquira and, was reported to be used in treatments of digestive complaints, colds, fevers, skin infections, inflammations and headaches (Table 4). Mentha ssp., introduced to the American continent, is widely popular in folk medicine $[9,35,44]$ and contribute to the popularity of introduced plant species.

\section{Popularity of plant families}

Popularity may also reflect world-wide presence of large plant families, such as the Lamiaceae. Contrary, Schippmann et al. [1], suggest that popularity of plant families is related to the local availability of the species. Studies in diverse localities as for example in Loja province, Ecuador [45], Imbituba, Santa Catarina, Brazil [35], Sierras de Córdoba, Argentina [46], and Banbungo, Cameroon [27] have found Asteraceae and Lamiaceae with high 
numbers of plant species used locally. These findings are comparatively similar to the results obtained by ethnobotanical studies carried out in the department of Boyacá, e.g. the studies of Lagos [9] in five municipalities in the central part of Boyacá, and a study carried out in the municipality of San José de Paré by Toscano [10]. The results indicate a large availability of species belonging to the Asteraceae and Lamiaceae in this region. At the same time, these results are comparable to the global pattern suggested by Moerman [47] who considered that Asteraceae and Lamiaceae are among the most used plant families in traditional medicine world-wide.

In relation to the popularity of Asteraceae, Garcia Barriga [48] reported that this family is the taxonomic group of plant species with the highest use in traditional medicine in Colombia. Nevertheless, the large range of distribution of this family may explain part of the popularity of this taxonomic group in folk medicine [27].

Furthermore, plant families, such as the Apiaceae, Apocynaceae and Guttiferae, of which there are examples of species with medicinal use in Campo Hermoso and Zetaquira, are part of the list of plant families with high numbers of traditionally used species worldwide [1].

However, $85 \%$ of the medicinal species identified in this study have been described and reported as medicinal in Colombia [11,48-50]. Of these species, $54 \%$ are introduced. This indicates that transmission of knowledge on introduced medicinal species has been successful in Colombia and in the studied municipalities. Similar situations have been observed and discussed in other countries as Brazil [28] where it was observed that knowledge of introduced plant species within farmers communities has been well established.

Promotion, conservation and sustainable use of native medicinal plant species may be facilitated by including shrubs and trees into local agroforestry systems. A number of these species, apart from being medicinal, offer also other kind of uses, e.g. as food, source of wood and shade. Such multiuse plants are often valuable to locals [31].

\section{Conclusions}

A total of 80 medicinal plant species were recorded. Of these, 78 species were taxonomically identified of which 35 were native species and 43 were introduced belonging to 74 genera and 41 floristic families. Among the native species $40 \%$ were found in natural habitats while twenty-seven per cent of introduced species were found as naturalised in the wild. The families with the highest numbers of species reported as medicinal were: Asteraceae, Lamiaceae, Apiaceae, Rutaceae, Verbenaceae, Malvaceae, Solanaceae and Urticaceae. Most applications of medicinal plants are related to diseases of the digestive and respiratory systems, and infections. Leaves are the most popular plant part used. Decoctions and oral administration are the most common practices.

The total of medicinal plants uses described by informants was higher in the more remote Campo Hermoso than in the more accessible Zetaquira. UVs of introduced plant species were significantly higher than native species in the more accessible municipality of Zetaquira, while there were no significant differences between the two groups in the more remote municipality of Campo Hermoso and nor in the two municipalities combined.

The list of the most popular medicinal plant species for healers and amateur healers respectively showed that $87 \%$ of the species from the amateur healers' list were introduced including mainly herbs while in the case of healers, $57 \%$ of the species of mainly trees and shrubs. Conversion of forests for agriculture and cattle ranching is depleting local forest resources and a number of medicinal plants were reported as disappearing or locally extinct. Only about $50 \%$ of the native medicinal species were found in cultivation. Agroforestry may provide an option for integrating agriculture with cultivation of native trees, shrubs and lianas, not otherwise cultivated. Educational programs could raise awareness in relation to conservation and maintaining use of native species.

\section{Consent}

Written informed consent was obtained from the patient for publication of this report and any accompanying images.

\section{Endnotes}

${ }^{a}$ Group of experts on the use of local medicinal plant species who offer local people treatments using medicinal plants.

${ }^{\mathrm{b}}$ Group of people who are interested in the use of medicinal plants, but do not work as professional healers.

${ }^{\mathrm{c}}$ Local term to refer to large home gardens with inclusion of a variety of trees and shrubs.

Competing interests

The authors declare that they have no competing interests.

\section{Authors' contributions}

Ana Lucia Cadena-González was the main responsible for study design, data analysis, interpretation, and writing. She conducted the field work in Campo Hermoso and Zetaquira between October 2008 and February 2009, collected the plant specimens, and identified the specimens in cooperation with the herbaria. Marten Sørensen and Ida Theilade contributed to the concept and design of the study, interpretation of the findings and preparation of the manuscript. All authors read and approved the final manuscript.

\section{Acknowledgements}

The authors acknowledge the collaboration of the local communities and authorities from the municipalities of Campo Hermoso and Zetaquira. We especially thank the interviewees and the participants in community meetings. The staff of the Colombian National Herbarium and of the Herbarium at Universidad Pedágogica y Tecnológica de Colombia is gratefully acknowledged for their help in the identification of the plant 
material. Finally, we thank DANIDA and the OTICON fund for the grants supporting the field work.

\section{Author details}

'Department of Agriculture and Ecology, University of Copenhagen, Rolighedsvej 21, 1958 Frederiksberg C, Denmark. ${ }^{2}$ Department of Plant and Environmental Sciences, University of Copenhagen, Rolighedsvej 21, 1958 Frederiksberg C, Denmark. ${ }^{3}$ Department of Food and Resource Economics, Faculty of Science, University of Copenhagen, Rolighedsvej 23, 1958 Frederiksberg C, Denmark.

Received: 7 May 2012 Accepted: 28 March 2013

Published: 11 April 2013

\section{References}

1. Schippmann U, Leaman DJ, Cunningham AB: Impact of cultivation and gathering of medicinal plants on biodiversity: global trends and issues, Biodiversity and the Ecosystem Approach in Agriculture, Forestry and Fisheries. Satellite event on the occasion of the Ninth Regular Session of the Commission on Genetic Resources for Food and Agriculture: 12-13 October 2002; Rome. FAO: FAO; 2002:1-21.

2. Muthu C, Ayyanar M, Rajan N, Ignacimuthu S: Medicinal plants used by traditional healers in Kaancheepuram District of Tamil Nadu. India. J Ethnobiol Ethnomed 2006, 2:43.

3. ProFound Advisors in Development: Market brief in the European Union for selected natural ingredients derived from native species. Crescentia cujete L., Calabash, Totumo. In Series of Market briefs on selected natural ingredients derived from native species in beneficiary countries of the BTFP. UNCTAD-United Nations Conference on Trade and Development, BioTrade Initiative/BioTrade Facilitation Programme (BTFP). Edited by United Nations. United Nations; 2005:1-40.

4. Dunmire WW: Gardens of New Spain. Austin: How Mediterranean plants and foods changed America. University of Texas Press; 2004

5. Quiroga R, Meneses L, Bussmann RW: Medicinal ethnobotany in Huacareta (Chuquisaca, Bolivia). J Ethnobiol Ethnomed 2012, 8:29. doi:10.1186/17464269-8-29.

6. Luziatelli G, Sørensen M, Theilade I, Mølgaard P: Asháninka medicinal plants: a case study from the native community of Bajo Quimiriki, Junín. Peru. J Ethnobiol Ethnomed 2010, 6:21

7. Dueñas M: A: Plan de desarrollo municipal 2008-2011. Alcaldía Municipal Campo Hermoso-Boyacá: La unidad de compromiso social; 2008.

8. Pacheco FP: Plan de Desarrollo Municipal de Zetaquira Boyacá 2004-2007. Desarrollo Municipal con Participación Ciudadana: Alcaldía Municipal Zetaquira- Boyacá; 2004.

9. Lagos LM: Especies con propiedades medicinales encontradas en las colecciones del herbario UPTC y su uso actual en Tunja y en los municipios de Chíquiza, Cómbita, Oicatá, Sora y Soracá (Boyacá). Revista De La Asociación Colombiana De Herbarios. Universidad del Quindío 2005, 25:58-68.

10. Toscano GJY: Uso tradicional de plantas medicinales en la vereda San Isidro, municipio de San José de Paré-Boyacá: Un estudio preliminar usando técnicas cuantitativas. Acta Biológica Colombiana 2006, 11:137-146.

11. Perez AE: Plantas Útiles de Colombia. Tercera redacción. Bogotá: Litografía Arco; 1956.

12. Cunningham AB: Applied Ethnobotany - People, Wild Plant Use and Conservation. London: Earthscan Publications; 2002.

13. Martin GJ: Ethnobotany, A methods manual. A People and Plants Conservation Manual. London: Chapman and Hall; 2004.

14. Thomas E, Vandebroek I, Van Damme P: What Works in the Field? A Comparison of Different Interviewing Methods in Ethnobotany with Special Reference to the Use of Photograhs. Econ Bot 2007, 61:376-384.

15. Colombian Amazonian Herbarium (COAH). www.sinchi.org.co/herbariov.

16. Dytham C: Choosing and Using Statistics - A Biologist's Guide. Oxford: Blackwell Sciences; 1999

17. Quinn GP, Keough MJ: Experimental Design and Data Analysis for Biologists. Cambridge: Cambridge University Press; 2002.

18. Sánchez M, Duivenvoorden JF, Duque A, Miraña P, Cavelier J: A Stem-based Ethnobotanical Quantification of Potential Rain Forest Use by Mirañas in north west Amazonia. Ethnobot Res Appl 2005, 3:215-229.
19. Phillips O, Gentry A: The Useful Plants of Tambopata, Peru: I. Statistical Hypotheses Tests With a New Quantitative Technique. Econ Bot 1993, 47:15-32.

20. Hoffman B, Gallaher T: Importance Indices in Ethnobotany. Ethnobot Res App/ 2007, 5:201-218.

21. Mc Cormick WP, Lyons NI, Hutcheson K: Distributional properties of Jaccard's Index of similarity. Commun Stat-Theor M 1992, 21:51-68.

22. Campos MT, Ehringhaus C: Plant Virtues are in the eyes of the beholders: A comparison of Known Palm Uses among Indigenous and Folk Communities of South-Western Amazonian. Econ Bot 2003, 57:324-344.

23. Ministerio de Protección Social, Colombia: Vademecum colombiano de plantas medicinales. Bogotá, D.C: El Ministerio; 2008

24. World Health Organization: Monographs on selected medicinal plants. Geneva, Switzerland: WHO Press; 2009.

25. Estupiñan GA, Jiménez EN: Uso de las plantas por grupos campesinos en la franja tropical del Parque Nacional Natural Paramillo (Córdoba, Colombia). Caldasia 2010, 32:21-32.

26. Ladio AH, Lozada M, Weigandt M: Comparison of traditional wild plant knowledge between aboriginal communities inhabiting arid and forest environments in Patagonia, Argentina. J Arid Environ 2007, 69:695-715.

27. Simbo DJ: An Ethnobotanical Survey of medicinal plants in Babungo, Northwest Region. Cameroon. J Ethnobiol Ethnomed 2010, 6:8.

28. De Almeida CC, Branco R, Ramos AM, De Amorin C, De Albuquerque UP: A comparison of knowledge about medicinal plants for three rural communities in the semi-arid region of north-east of Brazil. J Ethnopharmacol 2010, 127:274-284.

29. De Albuquerque UP: Re-examining hypotheses concerning the use and knowledge of medicinal plants: a study in the Caatinga vegetation of NE Brazil. J Ethnobiol Ethnomed 2006, 2:30.

30. Theilade I, Hansen HH, Krog MP: Ethnobotanical knowledge: implications for participatory forest management. J Transdiscipl Environ Stud 2007, 6:1-14

31. Fonnegra GR: Antecedentes Para la Construcción de una Farmacopea ó el Vademecum Colombiano de Plantas Medicinales. In Corpochivor, Instituto Humboldt, Universidad Pedagógica y Tecnológica de Colombia. Universidad Pedagógica y Tecnológica de Colombia: Universidad Distrital Francisco José de Caldas; 2006:101-160.

32. Njoroge GN, Joroge GN, Kaibui IM, Njenga PK, Odhiambo PO: Utilisation of priority traditional medicinal plants and local people's knowledge on their conservation status in arid lands of Kenya (Mwingi District). J Ethnobiol Ethnomed 2010, 6:22.

33. CRAF-PROSEA network: World Agroforestry Centre, Agroforestry Tree Database. http://www.worldagroforestry.org/treedb2/AFTPDFS/Carissa_edulis.pdf.

34. Secretaria de Salud de Boyacá: Análisis Situacional del Municipio de Zetaquira. Boyacá: Dirección Técnica de Salud Pública, Vigilancia en Salud Pública; 2008

35. Zank S, Hanazaki N: Exploring the links between Ethnobotany, Local Therapeutic Practices, and Protected Areas in Santa Catarina Coastline. Evid Based Complement Alternat Med: Brazil; 2011. doi:10.1155/2012/563570.

36. Neves JM, Matos C, Mouthinho C, Queiroz G, Gomes Lígia Rebelo G: thnopharmacological notes about ancient uses of medicinal plants in Trás-os-Montes (northern of Portugal). J Ethnopharmacol 2009, 124:270-283.

37. De Albuquerque UP, Da Silva VA, Cabral M, Alencar NL, Andrade L: Comparisons between the use of medicinal plants in indigenous and rural caatinga (dryland) communities in NE Brazil. Press Boletin Latinoamericano y del Caribe de Plantas Medicinales y Aromaticas 2008, 7:156-170.

38. Merlin F, Narasimham D: Plant names and uses as indicators of knowledge patterns. Indian J Tradit Know 2009, 8:645-648.

39. Ladio AH, Lozada M: Comparison of wild edible plant diversity and foraging strategies in two aboriginal communities of northwestern Patagonia. Biodivers Conserv 2003, 12:937-951.

40. Case RJ, Pauli GF, Soejarto DD: Factors in maintaining indigenous knowledge among ethnic communities of Manus Island. Econ Bot 2005, 59:356-365.

41. Johnson E, Emche S: Variation of Alkaloid Content in Erythroxylum coca Leaves from Leaf Bud to Leaf Drop. Ann Bot-London 1994, 73:645-650

42. Barni M, Fontanals A, Moreno S: Estudio de la eficacia antibiótica de un extracto etanólico de Rosmarinus officinalis L. contra Staphylococcus aureus en dos modelos de infección en piel de ratón. [Study of the antibiotic efficacy of an ethanolic extract from Rosmarinus officinalis 
against Staphylococcus aureus in two skin infection models in mice]. Boletin Latinoamericano y del Caribe de Plantas Medicinales y Aromáticas 2009, 8:219.

43. Rojas $L B$, Usubillaga A: Composition of the essential oil of Satureja brownei (S.W.) Briq. from Venezuela. Flavour Frag J 2000, 15:21-22.

44. Gomez H, Díaz F, Franco L, Mercado J, Guzmán J, Medina J, Gaitán R: Folk medicine in the northern coast of Colombia: an overview. J Ethnobiol Ethnomed 2011, 7:27.

45. Morocho V, Tinitana F, Malagón O, Zaragoza T, Armijos C, Medina M, Medina I, Chalán D, Lojan C, Lavanda C, Minga P, Cartuche J: Estudio Etnobotánico de especies medicinales en la comunidad indígena "Saraguro" de la Provincia de Loja, Ecuador. In Proocedings of III National Conference in Medicinal and Aromatic Plants, and Tropical Floriculture: 26-27 October 2006; Municipality of Jenesano, Boyacá. Edited by Corpochivor: Instituto Humboldt. Universidad Distrital Francisco José de Caldas: Universidad Pedagógica y Tecnológica de Colombia; 2006:46-80.

46. Martínez GJ, Luján MC: Medicinal plants used for traditional Veterinary in the Sierras de Córdoba-Argentina: An ethnobotanical comparison with human medicinal uses. J Ethnobiol Ethnomed 2011, 7:23.

47. Moerman DE, Pemberton RW, Kiefer D, Berlin B: A comparative analysis of five medicinal floras. Journal of Ethnobiol 1999, 19:49-67.

48. García Barriga H: Flora Medicinal de Colombia, Tomos I-III. 2nd edition. Bogotá: Tercer Mundo Editores; 1992.

49. Pbro. Arias E: Plantas Medicinales. Vigésima Ediciónth edition. Medellín: La Pluma de Oro; 1991.

50. Fonnegra R, Jimenez SL: Plantas medicinales aprobadas en Colombia. 2nd edition. Medellín: Universidad de Antioquia; 2007.

doi:10.1186/1746-4269-9-23

Cite this article as: Cadena-González et al: Use and valuation of native and introduced medicinal plant species in Campo Hermoso and Zetaquira, Boyacá, Colombia. Journal of Ethnobiology and Ethnomedicine 2013 9:23.

\section{Submit your next manuscript to BioMed Central and take full advantage of:}

- Convenient online submission

- Thorough peer review

- No space constraints or color figure charges

- Immediate publication on acceptance

- Inclusion in PubMed, CAS, Scopus and Google Scholar

- Research which is freely available for redistribution 